\title{
Scalar imaging velocimetry measurements of the velocity gradient tensor field in turbulent flows. II. Experimental results
}

\author{
Lester K. Su and Werner J. A. Dahm ${ }^{\mathrm{a})}$ \\ Gas Dynamics Laboratories, Department of Aerospace Engineering, The University of Michigan, \\ Ann Arbor, Michigan 48109-2118
}

(Received 26 September 1995; accepted 22 March 1996)

\begin{abstract}
Scalar imaging velocimetry is here applied to experimental turbulent flow scalar field data to yield the first fully resolved, non-intrusive laboratory measurements of the spatio-temporal structure and dynamics of the full nine-component velocity gradient tensor field $\nabla \mathbf{u}(\mathbf{x}, t)$, as well as the pressure gradient field $\nabla p(\mathbf{x}, t)$, in a turbulent flow. Results are from turbulent flows at outer scale Reynolds numbers in the range $3,000 \leqslant \operatorname{Re}_{\delta} \leqslant 4,200$, with Taylor scale Reynolds numbers $\operatorname{Re}_{\lambda} \approx 45$. These give a previously inaccessible level of detailed experimental access to the spatial structure in the velocity gradient tensor field at the small scales of turbulent flows, and through the much longer temporal dimension of these four-dimensional data spaces allow access to the inertial range of scales as well. Sample spatio-temporal data planes and probability distributions spanning more than 75 advection time scales $\left(\lambda_{\nu} / U\right)$ are presented for various dynamical fields of interest, including the three components of the velocity field $\mathbf{u}(\mathbf{x}, t)$, the nine components of the velocity gradient tensor field $\nabla \mathbf{u}(\mathbf{x}, t)$ through the full vector vorticity field $\omega_{i}(\mathbf{x}, t)$ and tensor strain rate field $\varepsilon_{i j}(\mathbf{x}, t)$, the kinetic energy dissipation rate field $\Phi(\mathbf{x}, t) \equiv 2 \nu \varepsilon: \varepsilon(\mathbf{x}, t)$, the enstrophy field $\frac{1}{2} \omega \cdot \omega(\mathbf{x}, t)$, the enstrophy production rate field $\omega \cdot \varepsilon \cdot \omega(\mathbf{x}, t)$, and the pressure gradient field $\nabla p(\mathbf{x}, t)$. Continuity tests show agreement with the zero divergence requirement that exceeds the highest values reported from single-point, invasive, multi-probe measurements. Distributions of strain rate eigenvalues as well as alignments of the strain rate eigenvectors with both the vorticity and scalar gradient vectors are in agreement with DNS results, as are distributions of the measured helicity density fields $\mathbf{u} \cdot \omega(\mathbf{x}, t)$. Results obtained for the true kinetic energy dissipation rate field show good agreement, up to 14th-order, with previous inertial range structure function exponents measured by Anselmet et al. [J. Fluid Mech. 140, 63 (1984)] at much higher Reynolds numbers. In addition, probability distributions scaled on inner variables show good agreement among buoyant and non-buoyant turbulent flow cases, further suggesting that these results are largely indicative of the high Reynolds number state of the inner scales of fully developed turbulent flows. (C) 1996 American Institute of Physics. [S1070-6631(96)01707-2]
\end{abstract}

\section{INTRODUCTION}

The lack of a method for measuring fully resolved, fourdimensional vector velocity fields $\mathbf{u}(\mathbf{x}, t)$ in turbulent flows has to date presented a fundamental obstacle to the study of their spatial structure and temporal dynamics, and thus to the development of models for the small scales of turbulent flows. Practical turbulent shear flows are characterized by enormous spatial and temporal complexity over a wide range of length scales, from the local outer scale $\delta$ to the local inner scale $\lambda_{\nu} \propto \delta \cdot \operatorname{Re}_{\delta}^{-3 / 4}$, where $\operatorname{Re}_{\delta}$ is the local outer scale Reynolds number $(u \delta / \nu)$ and $\delta$ and $u$ are the length and velocity scales characterizing the local mean shear in the flow. This complexity, and in particular the range of length and time scales over which it occurs, precludes any direct numerical simulations (DNS) of the full Navier-Stokes equations in high Reynolds number turbulent flows. Numerical simulations can then at best determine the fluid motion only at relatively large scales, but even this requires that the dynamical interactions between these large scale motions and the motion at smaller scales be modeled. Large eddy simulations (LES) of this type thus require subgrid scale models

${ }^{a}$ Corresponding author. Electronic mail: wdahm@engin.umich.edu which, at a minimum, properly reproduce the interscale energy transfer and other relevant dynamical interactions between the large and small scales in the underlying velocity gradient fields.

It is widely accepted that any general model of these interactions must be based on the physical structure and dynamics of the velocity gradient fields $\nabla \mathbf{u}(\mathbf{x}, t)$ over the full range of turbulent length scales. However, even in the case of incompressible turbulent flow, relatively little is known about the spatial structure and temporal dynamics of the velocity gradient fields. Laboratory experiments capable of directly yielding useful information on the detailed structure and dynamics of the velocity gradient fields at fine scales have been few. This has largely restricted investigations of the three-dimensional spatial structure of the full velocity gradient tensor in turbulent flows to direct numerical simulations of the full equations of fluid motion. Though such simulations have provided considerable insight, they remain largely limited to simple flows such as homogeneous isotropic or sheared turbulence, in periodic domains, at relatively low Reynolds numbers. In practice, these simulations also often omit certain physical features of real turbulent flows. In shear flow simulations, for example, the Reynolds numbers, and thus the achievable range of outer to inner scales, are 

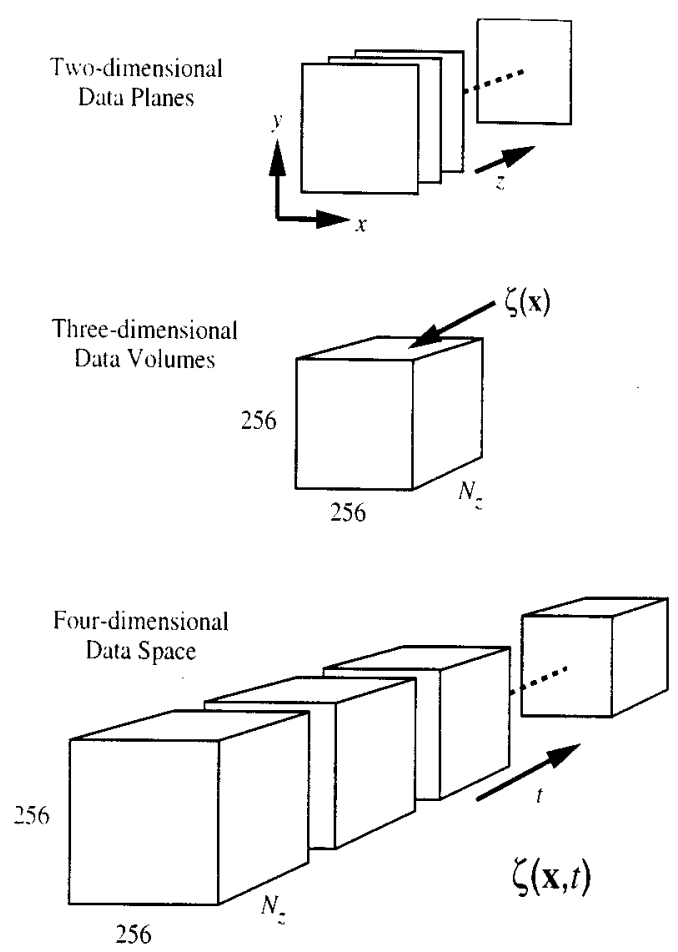

FIG. 1. Schematic showing the structure of the experimental scalar field measurements. A sequence of $N_{z}$ two-dimensional planes, parallel in the $z$-direction, forms the three-dimensional data volumes as shown. Measurement of these volumes is repeated in time to yield the final four-dimensional data space, which consists of over three billion individual point measurements of the scalar field.

restricted by computational limitations. Additionally, the difficulties in defining upstream and downstream boundary conditions have typically precluded rigorous simulations of spatially developing flows, with efforts instead being focused on time evolving flows, which may demonstrate very different large scale dynamics and entrainment properties. As a result of these experimental and computational limitations, the fine structure and dynamics of the velocity field $\mathbf{u}(\mathbf{x}, t)$, and in particular of the more dynamically insightful vorticity and strain rate fields $\omega(\mathbf{x}, t)$ and $\varepsilon(\mathbf{x}, t)$, in real, inhomogeneous, anisotropic turbulent shear flows at even moderately high outer scale Reynolds numbers have remained largely inaccessible to direct study.

Here we apply the scalar imaging velocimetry technique, developed in Ref. 1 and in a companion paper (Ref. 2 hereafter referred to as Part I), to obtain the first laboratory measurements of the space- and time-varying vector velocity field $\mathbf{u}(\mathbf{x}, t)$, as well as the full velocity gradient tensor field $\nabla \mathbf{u}(\mathbf{x}, t)$, in a turbulent shear flow. The scalar field data $\zeta(\mathbf{x}, t)$ used are the fully resolved, four-dimensional measurements of Southerland and Dahm. ${ }^{3,4}$ The resulting velocity gradient tensor fields offer a level of insight into the small scale structure and dynamics of turbulent flows which has previously been unavailable to experiments. Among the results presented are sample spatio-temporal data planes, as well as probability distributions, for the full vector vorticity field $\nabla \times \mathbf{u}(\mathbf{x}, t)$, the full tensor strain rate field $\varepsilon(\mathbf{x}, t)$, the enstrophy field $\frac{1}{2} \omega \cdot \omega(\mathbf{x}, t)$, the enstrophy production rate
TABLE I. Characteristics of the three data sets used in the present study, together with the spatial and temporal separations in the resulting fourdimensional scalar field data spaces. The data were collected in the far-field of an axisymmetric turbulent jet. $\operatorname{Re}_{\delta}$ and $\operatorname{Re}_{\lambda_{T}}$ are the outer scale and Taylor scale Reynolds numbers, respectively. $N_{z}$ is the number of $z$-planes in each three-dimensional data volume. The in-plane grid spacing is $\Delta x=\Delta y$, and the interplane spacing is $\Delta z$. The interplane temporal spacing $\Delta t$ is the time between adjacent measurement planes in the $z$-direction, while $\Delta T$ is the temporal spacing between successive measurements of $\zeta(\mathbf{x}, t)$ at the same spatial point.

\begin{tabular}{lccccccc}
\hline \hline Data set & $\operatorname{Re}_{\delta}$ & $\operatorname{Re}_{\lambda_{T}}$ & $N_{z}$ & $\Delta x, \Delta y(\mu \mathrm{m})$ & $\Delta z(\mu \mathrm{m})$ & $\Delta t(\mathrm{~ms})$ & $\Delta T(\mathrm{~ms})$ \\
\hline $\mathrm{R} 0420$ & 3,000 & 41 & 7 & 108 & 90 & 8.87 & 62.1 \\
$\mathrm{R} 0628$ & 3,200 & 42 & 6 & 116 & 120 & 8.87 & 53.2 \\
$\mathrm{R} 0811$ & 4,200 & 48 & 6 & 107 & 110 & 8.87 & 53.2 \\
\hline \hline
\end{tabular}

field $\omega \cdot \varepsilon \cdot \omega(\mathbf{x}, t)$, the kinetic energy dissipation rate field $\Phi(\mathbf{x}, t)$, and the pressure gradient field $\nabla p(\mathbf{x}, t)$. These measurements are also used to examine the local alignments of the vorticity and scalar gradient vector fields with the eigenvectors of the strain rate tensor field, as well as to investigate the helicity density distribution, and inertial range scaling exponents up to 16th-order. Collectively, these measurements provide a level of detailed access to the structure and dynamics of turbulent flows that has previously been available only through direct numerical simulations under simplified turbulence conditions.

The presentation is organized as follows. Section II gives a brief description of the scalar imaging measurements, focusing on the issues of resolution and differentiability of the measured scalar fields. The application of scalar imaging velocimetry to actual turbulent flow scalar field data is reported in Section III C; the results given in that section are used to investigate the detailed spatial structure, temporal dynamics and associated statistics of various dynamical fields at the small scales of turbulent flows. Concluding remarks are given in Section IV.

\section{SCALAR IMAGING MEASUREMENTS}

The scalar imaging velocimetry technique ${ }^{1,2}$ is motivated by the demonstrated experimental capability for obtaining fully resolved, four-dimensional measurements of the fine

TABLE II. Resolution characteristics of the experimental scalar field measurements. The strain-limited molecular diffusion scale $\lambda_{D}$ represents the finest length scale on which gradients can be sustained in the scalar field. The smallest scalar field temporal scale is given by $\lambda_{D} / U$, where $U$ is the mean streamwise velocity in the jet at the measurement location. The remaining quantities in the table are normalized with these reference scales. The quantities $\Delta x, \Delta y, \Delta z$ and $\Delta T$ are all less than $\sim 0.5$, indicating that the data are simultaneously differentiable in all three spatial dimensions and time. The in-plane temporal separations $\Delta t_{x}$ and $\Delta t_{y}$ demonstrate that the measurement of $\zeta$ at adjacent points in $x$ and $y$ is effectively simultaneous, while the significantly larger interplane spacing $\Delta t$ necessitates corrections in the method of $z$-differentiation of the data, as discussed in Section II B.

\begin{tabular}{cccccccc}
\hline \hline Data set & $\lambda_{D}(\mu \mathrm{m})$ & $\lambda_{D} / U(\mathrm{~ms})$ & $\Delta x, \Delta y$ & $\Delta z$ & $\Delta t_{x, y}$ & $\Delta t$ & $\Delta T$ \\
\hline R0420 & 303 & 152 & 0.356 & 0.297 & $<0.0003$ & 0.058 & 0.409 \\
R0628 & 289 & 136 & 0.401 & 0.415 & $<0.0003$ & 0.065 & 0.391 \\
R0811 & 239 & 85 & 0.447 & 0.460 & $<0.0005$ & 0.104 & 0.627 \\
\hline \hline
\end{tabular}



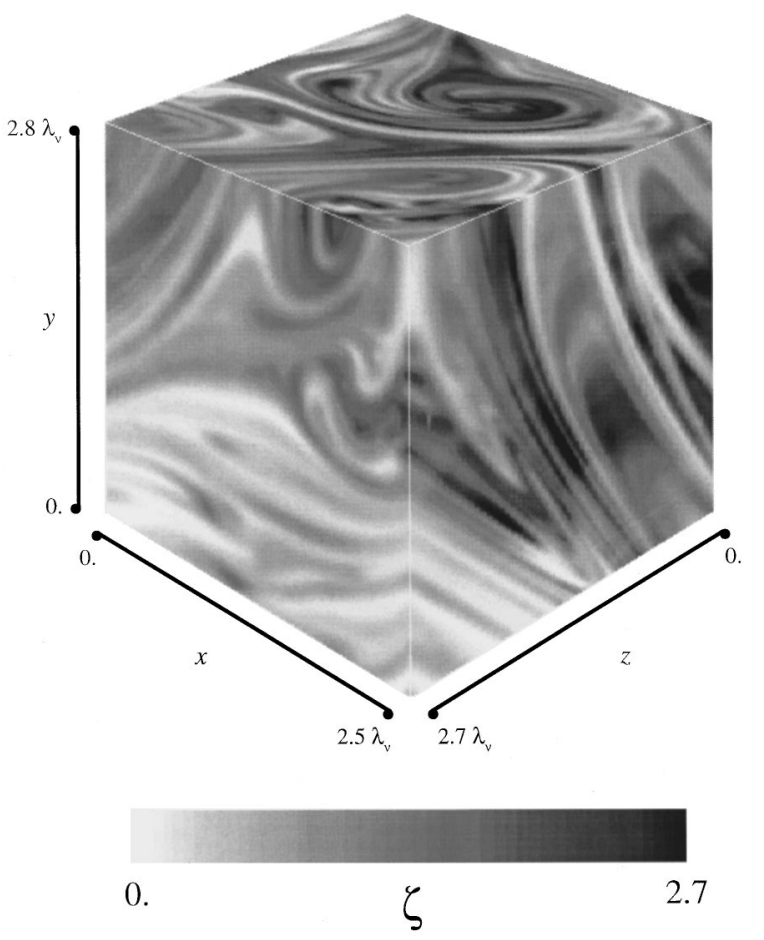

FIG. 2. An example of a three-dimensional data volume obtained using the scalar imaging diagnostic described in Section II A and Southerland and Dahm (Refs. 3 and 4). Shown here is the scalar field $\zeta(\mathbf{x}, t)$ from Case R0806. This full $256^{3}$ volume demonstrates the extent to which this measurement technique yields information on the three-dimensional spatial structure of the scalar field.

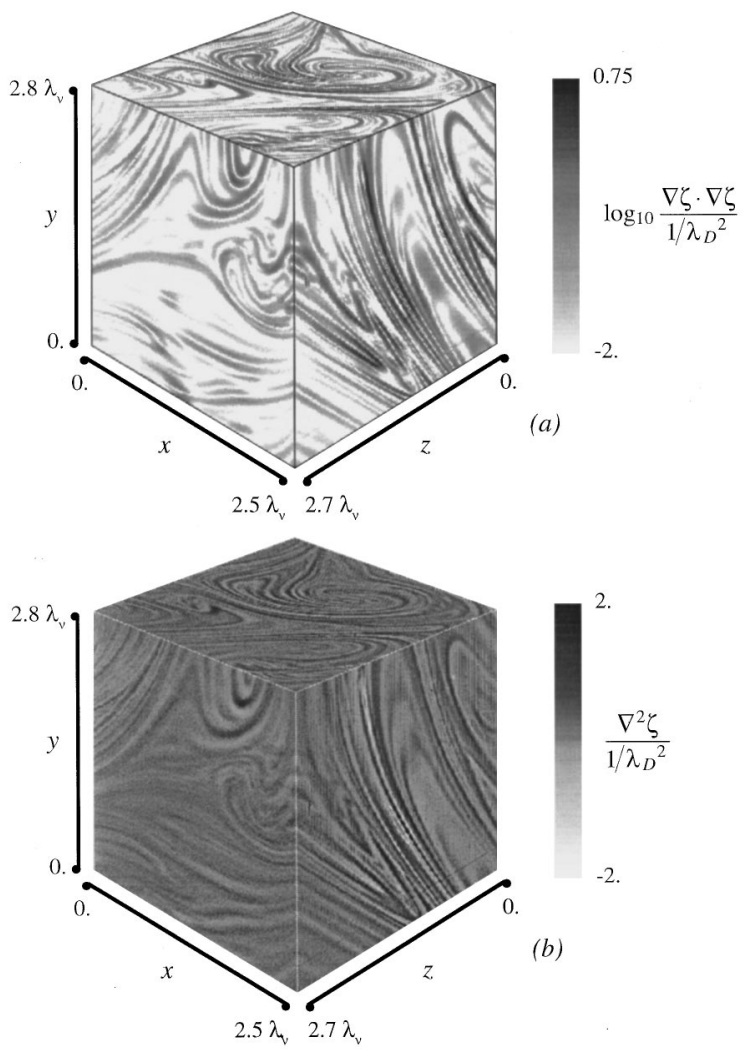

FIG. 3. (a) The scalar energy dissipation rate $\nabla \zeta \cdot \nabla \zeta$ and (b) the Laplacian $\nabla^{2} \zeta$ fields corresponding to the scalar field $\zeta(\mathbf{x}, t)$ shown in Fig. 2. These are obtained via central differencing on $\zeta(\mathbf{x}, t)$ and indicate the high resolution and signal quality attained by these measurements.

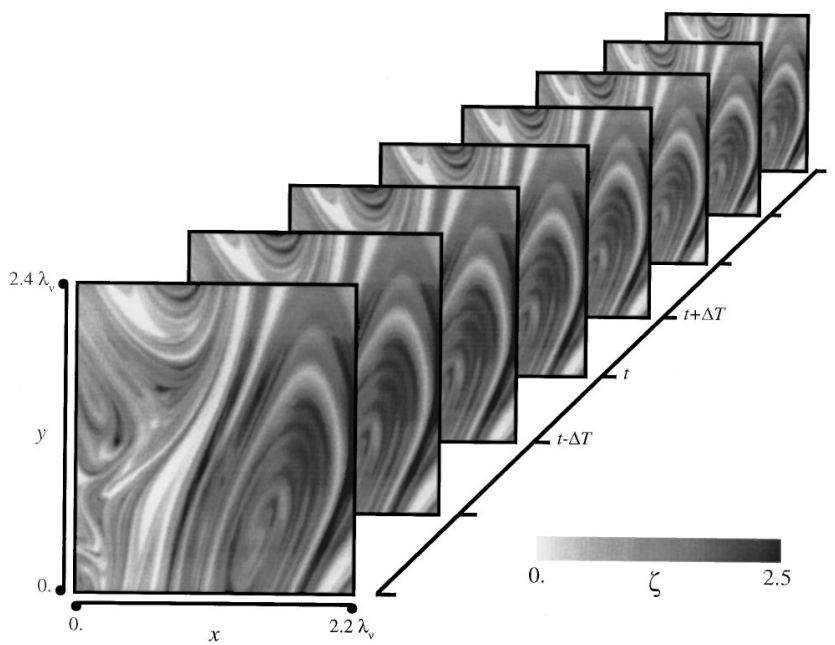

FIG. 4. The scalar field $\zeta(\mathbf{x}, t)$ at eight adjacent time steps, from the data set R0811 (Refs. 3 and 4). By reducing $N_{z}$, the number of $z$-planes per data volume in the measurement, the time step $\Delta T$ between these planes is kept sufficiently small to permit time differentiation of the scalar field.

scale structure of $\mathrm{Sc} \gg 1$ conserved scalar fields $\zeta(\mathbf{x}, t)$ on the inner scales of turbulent flows. ${ }^{3-5}$ The ability to determine the time derivative $\partial \zeta(\mathbf{x}, t) / \partial t$, scalar gradient $\nabla \zeta(\mathbf{x}, t)$, and Laplacian $\nabla^{2} \zeta(\mathbf{x}, t)$ fields from these fully resolved measurements leaves as the only unknowns in the exact conserved scalar transport equation the components of the velocity field $\mathbf{u}(\mathbf{x}, t)$. This section gives a brief description of the scalar

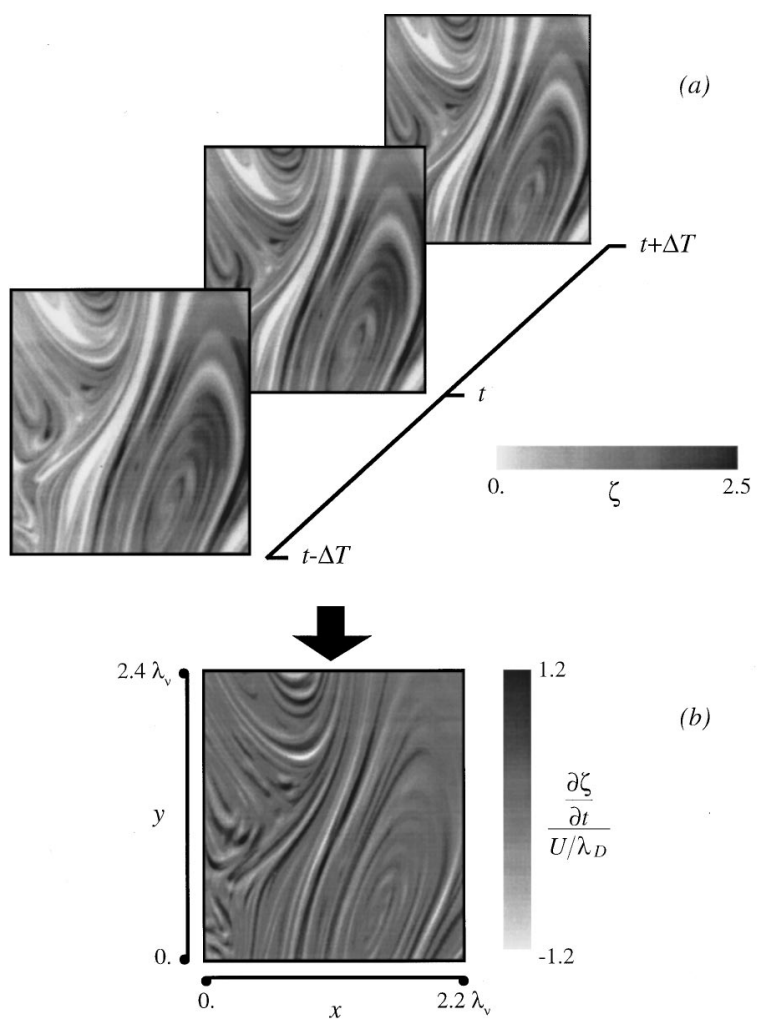

FIG. 5. (a) The scalar field $\zeta(\mathbf{x}, t)$ at three successive instants in time, from the time series of Fig. 4. Also shown (b) is the time derivative field $\partial \zeta(\mathbf{x}, t) / \partial t$ for the center plane. This time derivative is obtained through a central difference on the two adjacent planes shown. 


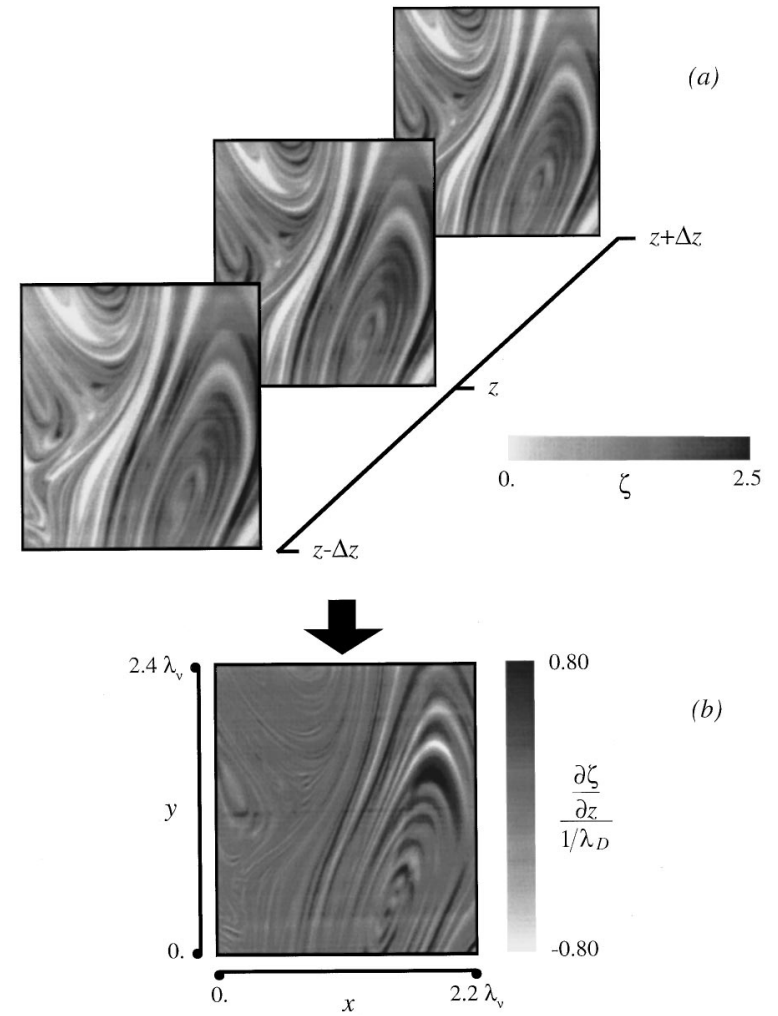

FIG. 6. (a) The scalar field $\zeta(\mathbf{x}, t)$ in three parallel $z$-planes, and (b) the $z$-derivative field $\partial \zeta(\mathbf{x}, t) / \partial t$ for the center plane. The center plane here is the same as that in Fig. 5. The $z$-derivative is calculated using information from the adjacent planes shown, and incorporates the time derivative information of Fig. 5 as described in Section II B.

field measurement technique, addresses the resolution and differentiability of the resulting data, and presents sample fully resolved, four-dimensional scalar field imaging results.

\section{A. Four-dimensional scalar field imaging}

The measurement technique and the resulting data used here have been described in detail in Southerland and Dahm. ${ }^{3-5}$ Briefly, the data are from laser induced fluorescence measurements of Sc $\gg 1$ scalar mixing in the selfsimilar far field of an axisymmetric turbulent jet in water. The concentration field $\zeta(\mathbf{x}, t)$ of the conserved, dynamically passive, laser fluorescent dye carried by the jet fluid is measured repeatedly in time at a large number of points within a small, three-dimensional spatial volume. The measurement location is 235 jet nozzle diameters $(1.15 \mathrm{~m})$ downstream of the jet exit and $13 \mathrm{~cm}$ off of the jet centerline. Each twodimensional measurement plane spans $2.5 \times 2.5 \mathrm{~cm}$, which may be compared with the local jet width $\delta=51 \mathrm{~cm}$. A schematic depicting the structure of the measured scalar fields is presented as Fig. 1. The imaging planes are formed by repeatedly sweeping a collimated laser beam in a raster fashion throughout the measurement volume, and the resulting fluorescence from the dyed jet fluid is imaged onto a twodimensional $256 \times 256$ high speed photodiode array. The beam is swept by a pair of low-inertia mirrors, which are driven by galvanometric scanners slaved to the imaging array timing. The array output was acquired serially at an 8-bit digital depth and continuously written in real time, at sus-

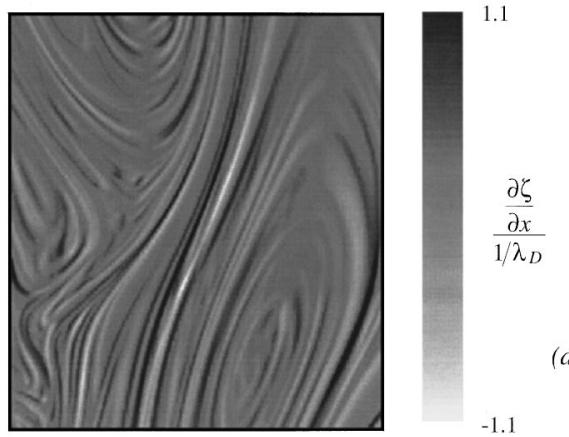

(a)

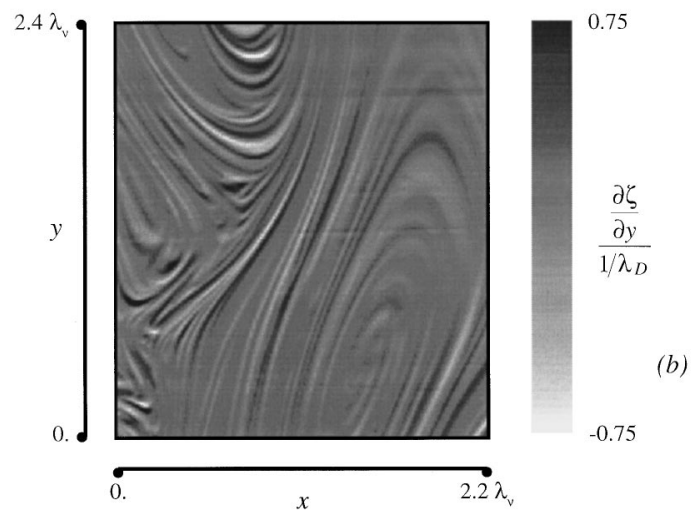

FIG. 7. The in-plane components of the scalar gradient field $\nabla \zeta$, for the center plane of Figs. 5 and 6. (a) The $\partial \zeta / \partial x$ component. (b) The $\partial \zeta / \partial y$ component.
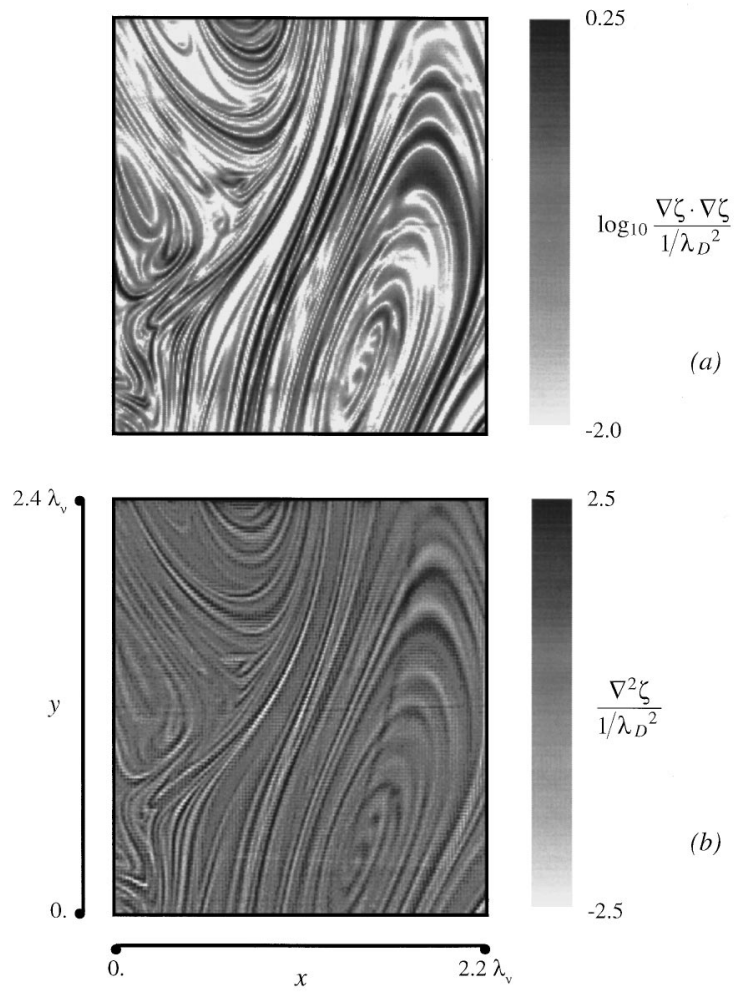

FIG. 8. (a) The scalar energy dissipation rate field $\nabla \zeta \cdot \nabla \zeta$ and (b) the Laplacian field $\nabla^{2} \zeta$ corresponding to the center plane of Figs. 5 and 6 . The time derivative field $\partial \zeta / \partial t$, the components of the scalar gradient field $\nabla \zeta$ and the Laplacian field $\nabla^{2} \zeta$ as shown in Figs. 5, 6, 7 and 8 provide the inputs to the scalar imaging velocimetry technique presented in Section III. 
tained rates of up to 142 planes/s, to a 3.1 gigabyte disk bank to produce the four-dimensional, spatio-temporal data structures as shown in Fig. 1. The resulting fluorescence intensity measurements are subsequently converted to the true dye concentration. Each measurement thus produces the scalar field $\zeta(\mathbf{x}, t)$ at over 3 billion points in space and time, representing in excess of 50,000 individual $256^{2}$ data planes. The specific parameters for the data used are given in Tables I and II.

\section{B. Resolution and differentiability}

All spatial and temporal differentiation of the experimental scalar field measurements is performed using linear central difference operators. Quantification of the resolution characteristics of these four-dimensional scalar field measurements is made in terms of reference length and time scales $l^{*}$ and $t^{*}$. We use for these reference scales the molecular diffusion length scale $\lambda_{D}=\lambda_{\nu} \cdot \mathrm{Sc}^{1 / 2}$ (Refs. 3, 6) and the local molecular diffusion scale advection time $\lambda_{D} / U$, which represent respectively the finest spatial and temporal length scales which can be sustained in the scalar field. Here $\lambda_{\nu} \approx 11.2 \cdot \delta \cdot \mathrm{Re}_{\delta}^{-3 / 4}$ is the finest viscous diffusion length scale, and $U$ is the local mean streamwise velocity. The resulting scaled resolution estimates $\Delta x, \Delta y, \Delta z$ and $\Delta T$ are given in Table II. The spatial separations $\Delta x, \Delta y, \Delta z$ are all less than 0.5 , indicating that the data are Nyquist sampled in space. This, combined with the high signal quality attained, allows accurate differentiation in all three spatial directions to obtain the true scalar gradient vector field $\nabla \zeta(\mathbf{x}, t)$. Similarly, the temporal separation $\Delta T$ between the same spatial point in successive data volumes is in all cases on the order of 0.5 , which indicates that the data can be differentiated in time as well.

However, the nature of these measurements, in which the parallel $(z-)$ planes are measured sequentially in time, means that the accuracy of the $z$-derivatives is affected not only by the grid spacing $\Delta z$ in that direction, but also by the temporal displacement $\Delta t_{z}$ between measurements of parallel planes. Where the value of $\Delta t_{x_{i}}$, the time between measurements of points spatially adjacent in the $x_{i}$ direction, begins to approach $\Delta x_{i}$, the accuracy of spatial derivatives computed using straight central differences may be called into question.

For points which are spatially adjacent in the $x$ - or $y$-directions, $\Delta t_{x_{i}}$ is manifestly far smaller than $\Delta x$ or $\Delta y$, as given in Table II, and central differencing is used without hesitation to determine $\partial \zeta / \partial x$ and $\partial \zeta / \partial y$. The measurements of $\zeta$ at points which are adjacent in $z$ are, however, separated temporally by the time necessary to measure $\zeta$ at $256^{2}$ points. From Table II, the scaled $\Delta t_{z}$ may then be as high as $0.2 \Delta z$ (in the case of the R0811 measurements). To quantify this effect, consider first the Taylor series representation of $\zeta$, for points separated in $z$ with no temporal separation (subscripts denote partial derivatives), namely

$$
\begin{aligned}
\zeta(z+\Delta z, t)= & \zeta(z, t)+\zeta_{z}(z, t) \cdot \Delta z+\frac{1}{2} \zeta_{z z}(z, t) \cdot \Delta z^{2} \\
& +O\left(\Delta z^{3}\right) .
\end{aligned}
$$

The central difference operator for $\partial \zeta / \partial z$ follows, as

$$
\frac{\partial \zeta}{\partial z}=\frac{\zeta(z+\Delta z, t)-\zeta(z-\Delta z, t)}{2 \Delta z}+O\left(\Delta z^{2}\right),
$$

which is second-order accurate. For these measurements, where the $z$-adjacent points are separated temporally by $\Delta t$ (this $\Delta t \equiv \Delta t_{z}$ from above), the Taylor expansion becomes

$$
\begin{aligned}
\zeta(z+ & \Delta z, t+\Delta t) \\
= & \zeta(z, t)+\zeta_{t}(z, t) \cdot \Delta t+\zeta_{z}(z, t) \cdot \Delta z+\zeta_{z t}(z, t) \cdot \Delta z \Delta t \\
& +\frac{1}{2} \zeta_{t t}(z, t) \cdot \Delta t^{2}+\frac{1}{2} \zeta_{z z}(z, t) \cdot \Delta z^{2}+O\left(\Delta^{3}\right) .
\end{aligned}
$$

Here the notation $O\left(\Delta^{3}\right)$ means that the remaining terms are third-order in any combinations of $\Delta t$ and $\Delta z$. The presence of the first-order term in $\Delta t$ means that the simple central difference expression of Eq. (2), if applied to these spatially and temporally separated points, would contain errors of the order $\Delta t / \Delta z$. However, the first-order term in $\Delta t$ involves the time derivative $\partial \zeta / \partial t$, which is itself determined to second-order accuracy using central differencing. Incorporating this information, we have the corrected operator

$$
\begin{aligned}
\frac{\partial \zeta}{\partial z}= & \frac{\zeta(z+\Delta z, t+\Delta t)-\zeta(z-\Delta z, t-\Delta t)-2 \zeta_{t}(z, t) \cdot \Delta t}{2 \Delta z} \\
& +O\left(\Delta^{2}\right),
\end{aligned}
$$

where the time derivative term $\zeta_{t}(z, t)$ which appears is the second-order estimate found using central differencing in time. By using the time derivative information in this fashion in determining the $z$ - spatial derivative, the errors which would arise from a naive application of the direct central difference operator (2) are avoided, and all of the components of the scalar gradient vector $\nabla \zeta$ are determined to an equivalent level of accuracy.

\section{Representative scalar field measurement results}

Figures 2 to 8 give representative results from this scalar field imaging diagnostic. A more extensive set of figures can be found in Refs. 3 and 4 . Figure 2 shows the measured scalar field $\zeta(\mathbf{x}, t)$ on a full $256^{3}$ data volume; Fig. 3 shows the resulting scalar energy dissipation rate field $\nabla \zeta \cdot \nabla \zeta(\mathbf{x}, t)$ and the Laplacian field $\nabla^{2} \zeta(\mathbf{x}, t)$ for the same volume. These volumes are taken from the data set labeled R0806 in Refs. 3 and 4, for which the jet outer scale Reynolds number was 5,000 . While the temporal spacing between these $256^{3}$ volumes is too great to allow for time differentiation, Figs. 2 and 3 give a good indication of the access to the threedimensional fine scale structure of the scalar field which this diagnostic provides.

The temporal resolution achievable by the fourdimensional measurements can be seen in Fig. 4, which shows a time series of the scalar field in the same plane from eight successive spatial volumes. Figure 5 shows a time series of three scalar field data planes from the R0811 case, together with the time derivative field corresponding to the center plane. The calculation of the time derivative for the center plane is made using a central difference, involving the two adjacent planes shown. Figure 6 shows a series of parallel scalar field data planes in $z$; the center plane here is the same as for the time series of Fig. 5. The $z$-derivative 

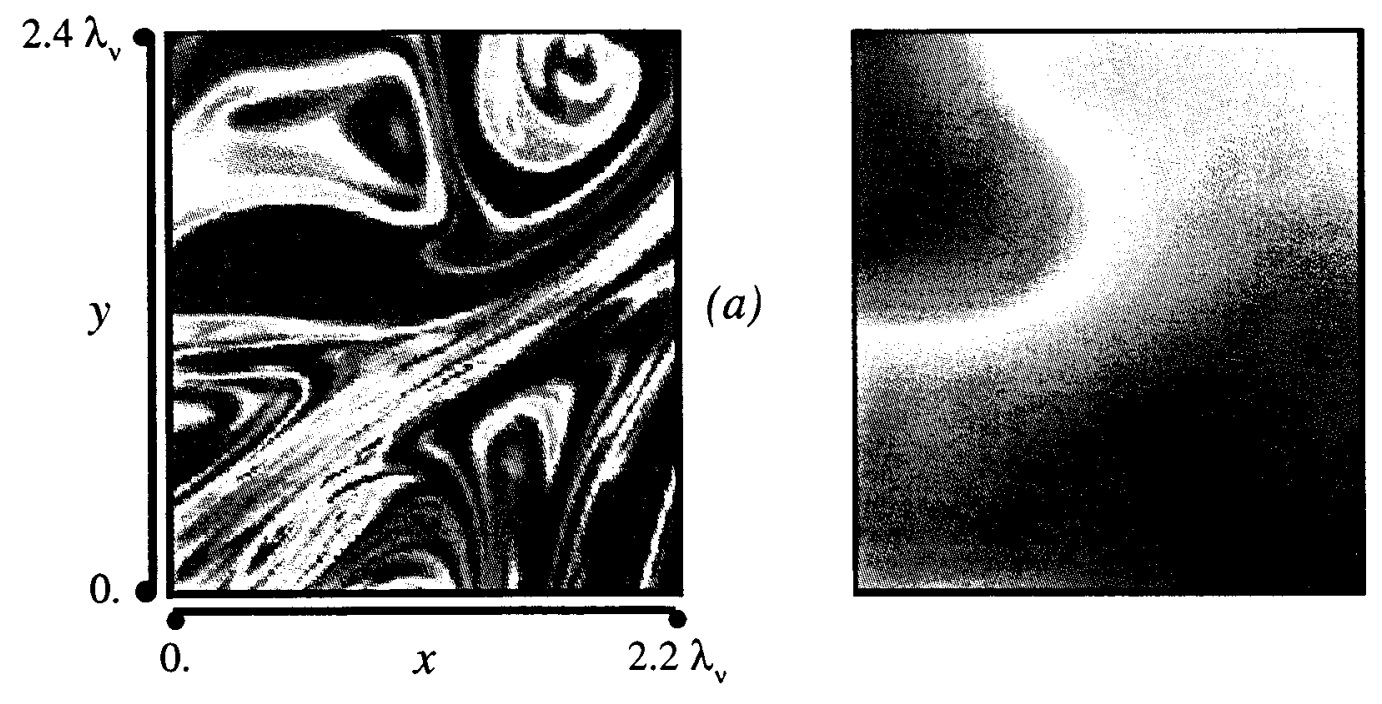

(b)
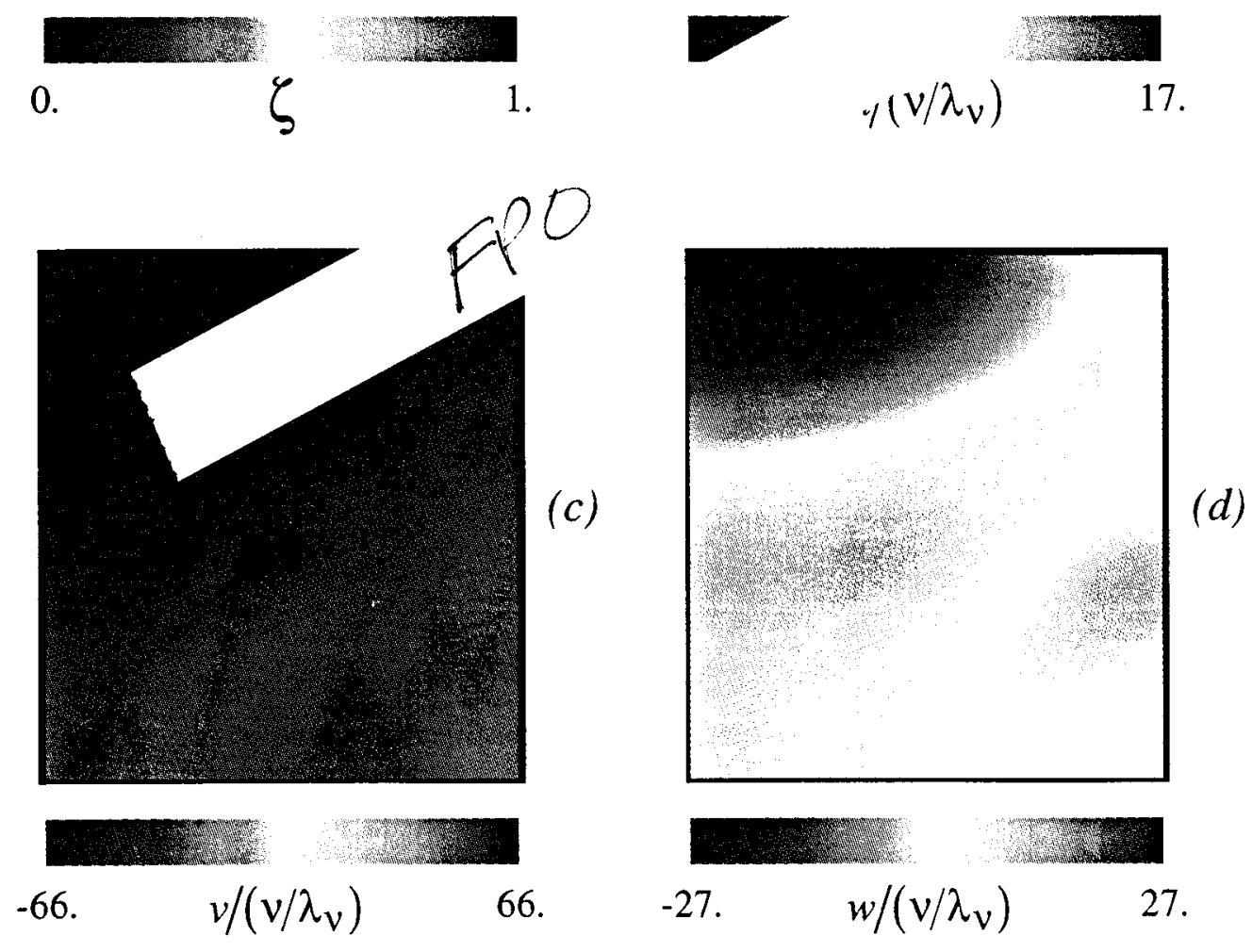

FIG. 9. A sample scalar field data plane (a) from the set R0811. This data set was collected in the far field of a turbulent jet, with outer scale Reynolds number $\operatorname{Re}_{\delta}=4200$. Also shown are the velocity component fields for this plane, found by integral minimization scalar imaging velocimetry as described in Section III B. The negative $y$-axis here corresponds to the streamwise direction in the jet. (b) The $u$-component. (c) The $v$-component. (d) The $w$-component.

$\partial \zeta / \partial z$ for the center plane is also shown in Fig. 6 and is determined from the adjacent $z$-planes shown, incorporating the time derivative information of Fig. 5 via Eq. (4). Finally, Fig. 7 gives the spatial derivative component fields $\partial \zeta / \partial x$ and $\partial \zeta / \partial y$ for the central plane of Figs. 5 and 6, and Fig. 8 shows the corresponding scalar energy dissipation rate and Laplacian fields, $\nabla \zeta \cdot \nabla \zeta$ and $\nabla^{2} \zeta$. These results give an indication of the resolution and differentiability achievable by scalar field measurements of this type. These measurements provide the input to the scalar imaging velocimetry method. ${ }^{1,2}$

\section{SCALAR IMAGING VELOCIMETRY RESULTS}

This section presents fully resolved, four-dimensional measurements of the structure and dynamics of the complete velocity gradient tensor field $\nabla \mathbf{u}(\mathbf{x}, t)$ in turbulent flows. These results are obtained by applying the integral minimization scalar imaging velocimetry approach of Part I to fully resolved turbulent scalar field data of the type described in Section II. The $\mathrm{Sc} \approx 2075$ scalar field data $\zeta(\mathbf{x}, t)$ used here are the three data sets of Refs. 3 and 4 labeled R0420, R0628 and R0811, for which the characteristics are given in Tables 


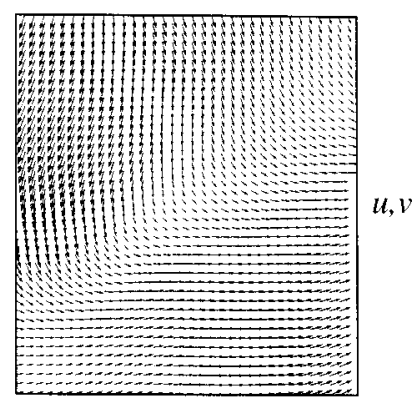

(a)

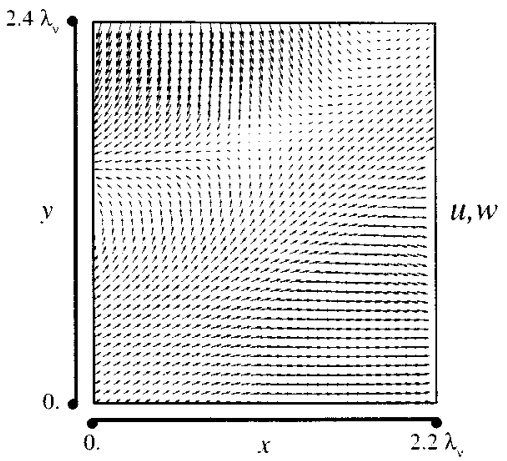

(b)

FIG. 10. Velocity vector projections, for the velocity component results of Fig. 9. Shown are the $u$-, $v$-projection (a) and the $u$-, w-projection (b). The mean streamwise velocity has been subtracted from the $v$-component, to emphasize the structure of the flow field.

I and II. The data are fully resolved in space and time, allowing direct differentiation to obtain $\partial \zeta(\mathbf{x}, t) / \partial t, \nabla \zeta(\mathbf{x}, t)$ and $\nabla^{2} \zeta(\mathbf{x}, t)$, as demonstrated in Section II. These derivative fields provide the inputs to the integral minimization SIV technique, which yields the underlying velocity field $\mathbf{u}(\mathbf{x}, t)$.

Section III A presents a brief review of the integral minimization scalar imaging velocimetry technique of Part I. Section III B describes the choice of parameters in the scalar imaging velocimetry inversion for the particular characteristics and structure of these data sets. Sections III C and III D present the $\mathbf{u}(\mathbf{x}, t)$ and $\nabla \mathbf{u}(\mathbf{x}, t)$ results for the three data sets, giving sample data planes as well as probability density functions over all of the data. The ability of the SIV technique to determine all three components of the velocity vector $\mathbf{u}(\mathbf{x}, t)$ and all nine components of the velocity gradient tensor $\nabla \mathbf{u}(\mathbf{x}, t)$ provides direct access to the structure and dynamics of the strain rate tensor field $\varepsilon(\mathbf{x}, t) \equiv \frac{1}{2}(\nabla \mathbf{u}$ $\left.+\nabla \mathbf{u}^{T}\right)$, and the vorticity vector field $\omega(\mathbf{x}, t) \equiv \nabla \times \mathbf{u}$. These results are given in Section III D 1. Following this, Section III D 2 examines the true kinetic energy dissipation rate fields $\Phi(\mathbf{x}, t) \equiv(2 / \operatorname{Re}) \varepsilon: \varepsilon$ and the enstrophy fields $\frac{1}{2} \omega \cdot \omega(\mathbf{x}, t)$, as well as the enstrophy production rate fields $\omega \cdot \varepsilon \cdot \omega(\mathbf{x}, t)$. In Section III D 3, comparisons are made with available data for the structure function exponents $\zeta_{q}$ giving the inertial range scalings for the velocity gradient fields, allowing for validation of the SIV results. The strain rate tensor eigenvalues are examined in Section III D 4, while Section III D 5 examines the alignments of the velocity vector, the vorticity vector and the eigenvectors of the strain rate tensor. The alignment of the scalar gradient vector $\nabla \zeta(\mathbf{x}, t)$ with the strain rate tensor eigenvectors is given in Section III D 6. This section concludes with a demonstration of the measurement of the pressure gradient field $\nabla p(\mathbf{x}, t)$ in sample planes in Section III D 7.

\section{A. Scalar imaging velocimetry}

The integral minimization formulation of scalar imaging velocimetry is described in detail in Part I. The problem of determining the velocity field $\mathbf{u}(\mathbf{x}, t)$ from the measured scalar field data $\zeta(\mathbf{x}, t)$ is through the minimization of the integral of a quantity $E$ over the measurement domain, where $E$ is a functional term dependent upon the components of the velocity vector $\mathbf{u}(\mathbf{x}, t)$ and the velocity gradient tensor $\nabla \mathbf{u}(\mathbf{x}, t)$. Formally,

$$
\min _{\mathbf{u}(\mathbf{x}) \in \mathfrak{R}^{3} \rightarrow \mathfrak{R}^{3}} \int_{D} E\left(u_{1}, u_{2}, u_{3} ; x_{1}, x_{2}, x_{3}\right) d^{3} \mathbf{x} .
$$

The components $u_{i}$ of $\mathbf{u}$ are the dependent variables in $E$, and the components $x_{i}$ of $\mathbf{x}$ are the independent variables. $E$ itself is written as

$$
E \equiv E_{1}+\alpha^{2} E_{2}+\beta^{2} E_{3}+\ldots,
$$

where each $E_{i} \geqslant 0$ represents a local residual involving the velocity field and possibly the scalar field. The factors $\alpha^{2}$, $\beta^{2}, \ldots>0$ allow control over the relative weights assigned to the individual terms $E_{i}$ in the minimization functional E.

Scalar imaging velocimetry is based on the exact conserved scalar transport equation, which in dimensionless form is

$$
\left[\frac{\partial}{\partial t}+\mathbf{u} \cdot \nabla-\frac{1}{\operatorname{Re~Sc}} \nabla^{2}\right] \zeta(\mathbf{x}, t)=0 .
$$

The residual $E_{1}$ in Eq. (6) is thus chosen to be the left-hand side of Eq. (7), and thus enforces the condition that the measured scalar field derivatives and any candidate velocity field $\mathbf{u}(\mathbf{x}, t)$ be in "good" agreement with the exact conserved scalar transport equation. Thus, formally

$$
E_{1} \equiv\left(\left[\frac{\partial}{\partial t}+\mathbf{u} \cdot \nabla-\frac{1}{\operatorname{ReSc}} \nabla^{2}\right] \zeta(\mathbf{x}, t)\right)^{2} .
$$

Because only incompressible turbulent flows will be considered, the second condition chosen is $\nabla \cdot \mathbf{u}=0$, and thus

$$
E_{2} \equiv(\nabla \cdot \mathbf{u})^{2} \text {. }
$$

The third condition chosen is one which measures the smoothness of the velocity field. Here we use a first-order Tikhonov stabilizer ${ }^{7}$

$$
E_{3} \equiv \nabla \mathbf{u}: \nabla \mathbf{u},
$$

which provides the solutions for $\mathbf{u}$ with the property of mathematical stability in the face of small noise or discretization errors in the initial data.

From the functional $E$ and its constituent terms $E_{1}, E_{2}$ and $E_{3}$, Eq. (5) can be solved for the components of $\mathbf{u}$. From the calculus of variations, the characteristic (Euler) equations which result for the three components $u, v, w$ are 


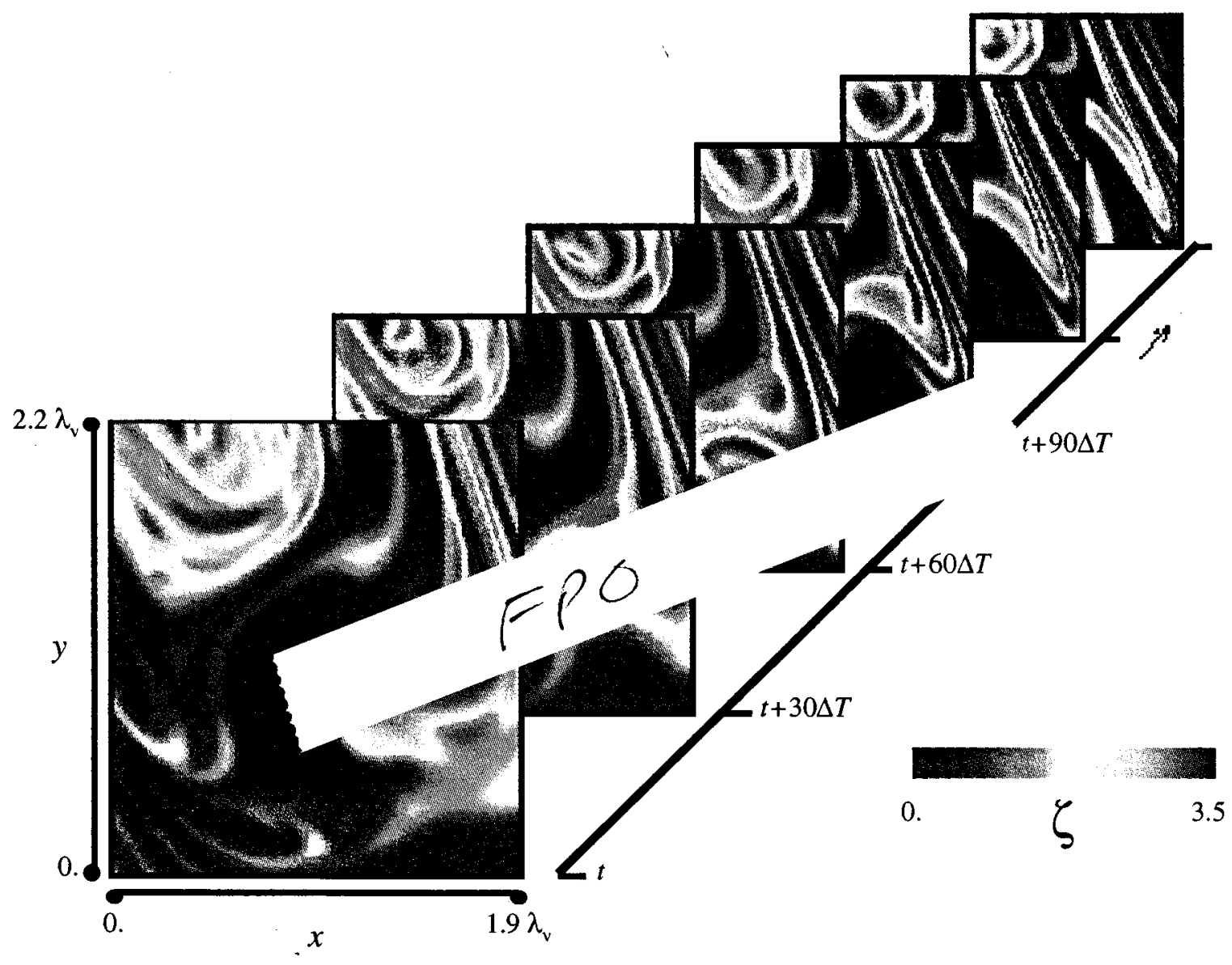

FIG. 11. A time series of six scalar field data planes from the data set R0628. The planes shown are separated in time by $30 \Delta T$, where $\Delta T$ is the separation between successive scalar field data volumes, and the velocity fields are determined for data volumes separated by $15 \Delta T$. The high Schmidt number of these measurements means that the velocity field will, in general, be time resolved and differentiable for the velocity field temporal separation $15 \Delta T$. Compare the clear time evolution of these scalar field planes with the small changes in the planes of Fig. 4, for which the scalar field is time differentiable.

$$
\begin{aligned}
u \zeta_{x}^{2} & +v \zeta_{y} \zeta_{x}+w \zeta_{z} \zeta_{x}-\alpha^{2}\left(u_{x x}+v_{y x}+w_{z x}\right)-\beta^{2} \nabla^{2} u \\
& =-\left(\frac{\partial \zeta}{\partial t}-\frac{1}{\operatorname{ReSc}} \nabla^{2} \zeta\right) \zeta_{x}
\end{aligned}
$$$$
u \zeta_{x} \zeta_{y}+v \zeta_{y}^{2}+w \zeta_{z} \zeta_{y}-\alpha^{2}\left(u_{x y}+v_{y y}+w_{z y}\right)-\beta^{2} \nabla^{2} v
$$$$
=-\left(\frac{\partial \zeta}{\partial t}-\frac{1}{\operatorname{ReSc}} \nabla^{2} \zeta\right) \zeta_{y}
$$$$
u \zeta_{x} \zeta_{z}+v \zeta_{y} \zeta_{z}+w \zeta_{z}^{2}-\alpha^{2}\left(u_{x z}+v_{y z}+w_{z z}\right)-\beta^{2} \nabla^{2} w
$$$$
=-\left(\frac{\partial \zeta}{\partial t}-\frac{1}{\operatorname{ReSc}} \nabla^{2} \zeta\right) \zeta_{z} \text {. }
$$

The velocity field $\mathbf{u}(\mathbf{x}, t)$ is then found by writing the above three equations for each of the discrete points in the measurement domain, representing the velocity derivative terms by discrete operators. Concatenating these equations results in a linear system which may be solved for the velocity field components.

\section{B. Application of the scalar imaging velocimetry technique}

The values for the weighting factors $\alpha^{2}$ and $\beta^{2}$, which weight the continuity and smoothness conditions, respectively, used in the DNS-based validation test in Part I were $\alpha^{2}=5 \cdot 10^{-4}$ and $\beta^{2}=2 \cdot 10^{-4}$ (though over a limited range of values the velocity field results were insensitive to the particular choices of $\alpha^{2}$ and $\beta^{2}$ ). The values of $\alpha^{2}$ and $\beta^{2}$ to be used here must reflect the much higher Schmidt number of these scalar field data. In addition, in the far-field of the turbulent jet the distribution of scalar values in the threedimensional data volume varies significantly with time. These fluctuations will result in variations in the magnitudes of the scalar field derivative terms in $E_{1}$ [defined by Eq. (8)], with the consequence that the effective weight on the scalar transport residual $E_{1}$ relative to $E_{2}$ and $E_{3}$ in Eq. (6) will change independent of the choices of $\alpha^{2}$ and $\beta^{2}$. To offset this, the scalar field values in each three-dimensional data volume are normalized by a uniform factor related to the peak scalar value. With this renormalization, the same values of $\alpha^{2}$ and $\beta^{2}$ can be used for all times $t$ in the measured 

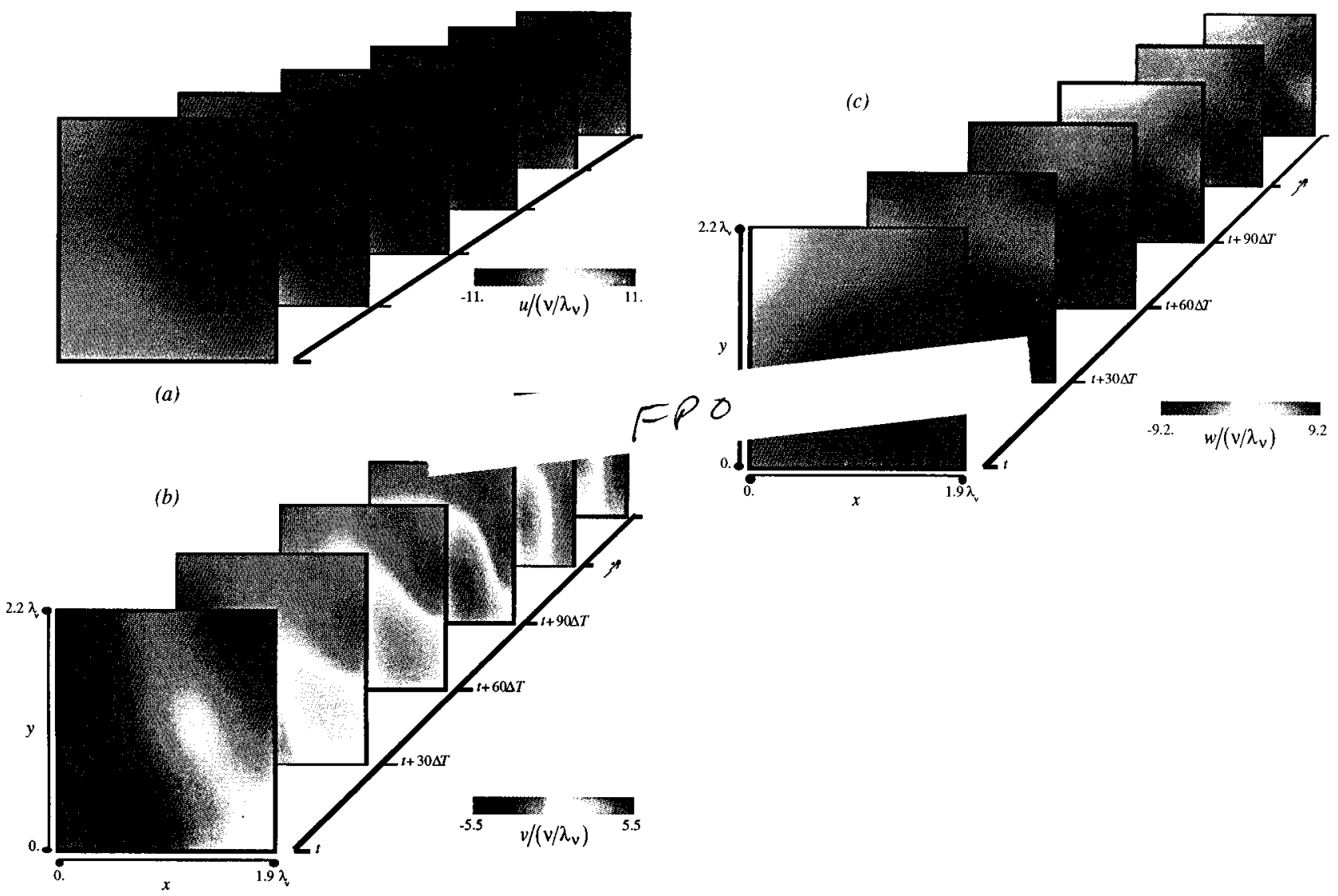

FIG. 12. The velocity component results for the scalar field time series of Fig. 11. Shown are (a) the $u$-component, (b) the $v$-component, and (c) the $w$-component.

scalar field data $\zeta(\mathbf{x}, t)$. Here the values used are $\alpha^{2}=0.72$ and $\beta^{2}=0.36$, which were found to yield a ratio of scalar to viscous length scales $\lambda_{D}$ and $\lambda_{\nu}$ consistent with the required $\lambda_{D} / \lambda_{\nu}=\mathrm{Sc}^{-1 / 2}$ in turbulent flows. ${ }^{1,2}$

Owing to the large Sc in these measurements, the scalar field contains far more information than the target velocity field. Proper subsampling of the scalar field data both in space and time can therefore reduce the total computational load required to perform the inversion, without compromising any information in the velocity field. For the present $\mathrm{Sc} \approx 2075$ scalar field data, the finest possible length scale $\lambda_{\nu}$ in the velocity field is roughly 45 times larger than the finest scale $\lambda_{D}$ in the scalar field data. However, the primary constraint on spatial subsampling of the data is not the resolution level of the velocity field, but rather is the structure of the scalar field data volumes. For each of the three data sets used, there are five usable planes in the $z$-direction. After central differencing there are three $z$-planes for which all of the scalar field derivative terms are known. Any spatial subsampling can thus only be performed in the $x-y$ plane. From Tables I and II, $\Delta x=\Delta y \approx \Delta z$ on the original measurement grid. Subsampling in the $x-y$ plane then results in a grid for which the in-plane grid spacing $\Delta x=\Delta y$ is larger than the interplane grid spacing $\Delta z$. Should this discrepancy in grid spacings become too large, the numerical difference operators, particularly those which represent mixed derivatives, may become unreliable. Experience has shown that a $4 \times$ subsampling in $x$-y does not have a noticeable effect on the results as compared with the original or $2 \times$ subsampled volumes. The nominal $64 \times 64 \times 3$ volumes which result have $1 / 16$ the number of points of the original volumes, which greatly increases the speed of the calculations.

The limited number of $z$-planes in these measurements becomes significant where the boundary conditions are concerned. Recall from Part I that the reflective boundary conditions used force $\partial u_{i} / \partial z=0$ at the $z$-boundaries of the spatial data volumes. Because of the proximity of these $z$-boundaries to the interior of the measurement volume, the $\partial u_{i} / \partial z$ values obtained throughout are inherently lower than the $\partial u_{i} / \partial x$ and $\partial u_{i} / \partial y$ values, for which the boundary conditions are imposed at a much greater distance. It is trivial to correct for this effect if to leading-order the true $z$-derivatives are taken to differ from their measured values by a multiplicative factor $\gamma$, which can then be readily found by matching the measured variance of $\partial w / \partial z$ to those of $\partial u / \partial x$ and $\partial v / \partial y$. The result is to increase the normal strain rate along $z$ by $\gamma$ while leaving the other normal strain rates unchanged, and to increase those shear strain rates and vorticity components involving $z$-derivatives by $2 \gamma /(\gamma+1)$, as has been done in the results presented in Sections III D 1 and III D 2.

In the same manner that the spatial resolution of the scalar field measurements results in over-resolution of the velocity field when both fields are viewed on the same grid, the time step $\Delta T$ of the measurements over-resolves the time 


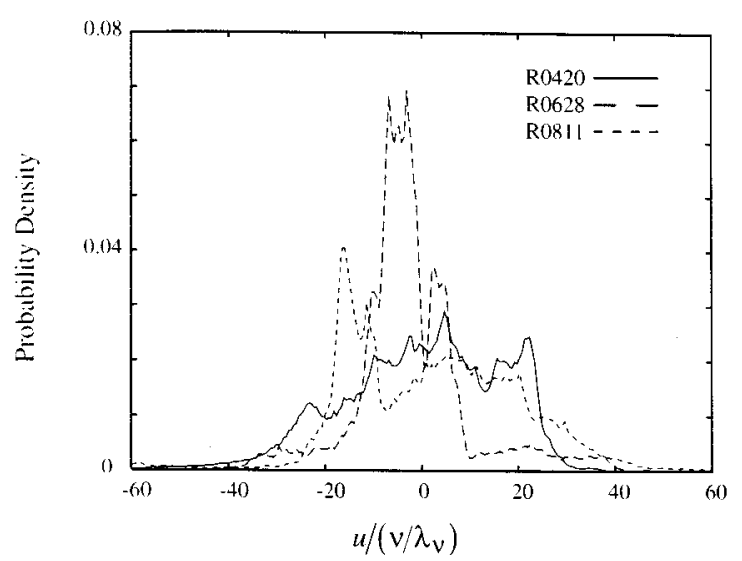

(a)

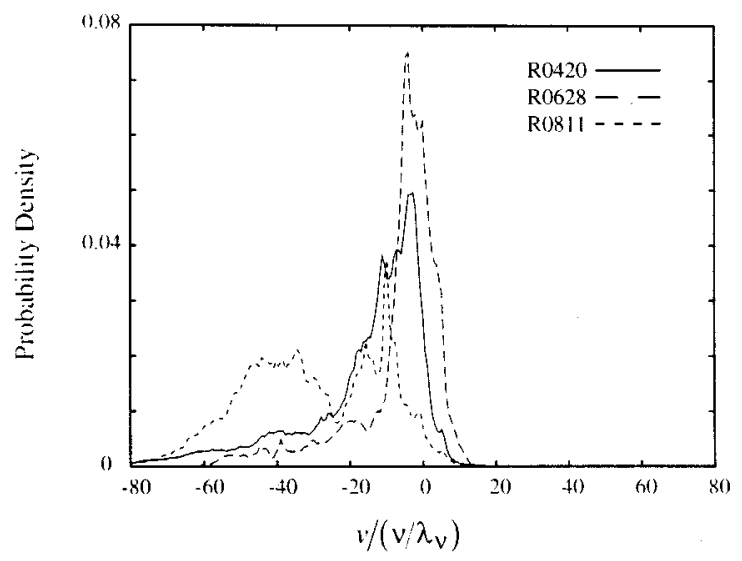

(b)

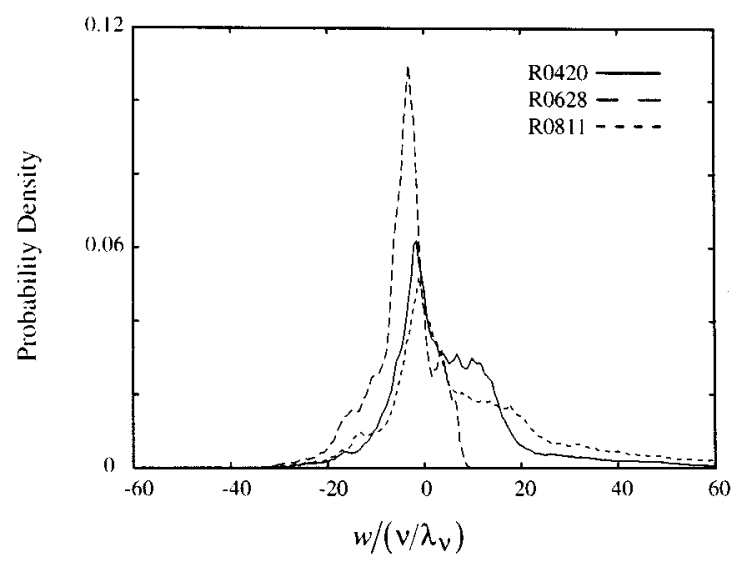

(c)

FIG. 13. Probability distributions of the velocity component values, for all time steps of the three data sets R0420, R0628 and R0811. (a) The $u$-component distribution, (b) the $v$-component distribution, and (c) the $w$-component distribution.

evolution of the velocity field. Defining a time scale for the velocity field in terms of the local advection velocity as $\lambda_{\nu} / U$, the temporal spacing $\Delta T$ in the scalar field data provides a level of time resolution of the velocity field roughly 45 times greater than the resolution of the scalar field. The choice here is to determine the velocity fields for scalar field times separated by $15 \Delta T$. For the R0420, R0628 and R0811 data sets there are, respectively, 5485, 5674 and 4266 scalar field time steps available, resulting in velocity fields for 365 , 378 and 284 time steps.

Because the turbulent jet in which these measurements of $\zeta(\mathbf{x}, t)$ are made entails the mixing of jet fluid with initial scalar concentration $\zeta_{0}$ with ambient fluid with zero scalar concentration, and because the scalar diffusivity is relatively small, there are substantial three-dimensional regions in the scalar field data volumes which have little or no scalar concentration. Such regions can be readily seen in, for example, Figs. 2 and 11. In these regions, the scalar field derivatives $\partial \zeta(\mathbf{x}, t) / \partial t, \nabla \zeta$, and $\nabla^{2} \zeta$ are negligibly small, resulting in the scalar transport condition $E_{1}$ being insignificant in relation to $E_{2}$ and $E_{3}$ in Eq. (6). For this reason, a criterion is used to exclude non-representative velocity and velocity gradient values when collecting statistics. This criterion is based on the scalar field time derivative $\partial \zeta(\mathbf{x}, t) / \partial t$. A given point $(\mathbf{x}, t)$ in the four-dimensional data volume is considered significant if $\partial \zeta(\mathbf{x}, t) / \partial t$ exceeds a certain threshold value, the threshold value used being dependent upon the noise level of the scalar field measurements. From Southerland, ${ }^{3}$ the rms noise level of the original 8-bit scalar field measurements is $\sim 1$ digital level; a simple error propagation analysis (e.g., Bevington ${ }^{8}$ ) on the central difference operator then yields an rms error in the time derivative of 1.4 (digital levels) $/ 2 \Delta T$. This error estimate is used as the threshold level on the scalar field time derivative. However, in identifying those points where the velocity component values are to be disregarded when collecting statistics, it would be overly restrictive simply to ignore all points where the time derivative falls below this threshold. Doing so would fail to recognize that the integral minimization scheme can deal with such regions of sparse scalar field information, provided that they are sufficiently small (as discussed in Refs. 9 and 10). If the characteristic length of these regions is not larger than the finest velocity gradient length scale $\lambda_{\nu}$, we can reasonably expect that the velocity field results in these regions will be accurate. The velocity component values at a given point $\mathbf{x}$ are thus ignored only if fewer than $10 \%$ of the points within $\lambda_{\nu} / 4$ of $\mathbf{x}$ have scalar time derivative values exceeding the chosen threshold. With this criterion, the $\mathbf{u}(\mathbf{x}, t)$ results at $86.6 \%$ of the original data points are used in collecting statistics.

\section{Sample spatial field results}

Figure 9 shows a scalar field plane from the data set R0811, together with the three velocity component fields in this plane as found by the SIV technique. Normalization of all variables here is performed with the inner variables $\nu$ and $\lambda_{\nu}$, and the resulting reference velocity $\nu / \lambda_{\nu}$. In the coordinate frame used, the increasing jet streamwise direction corresponds to the negative $y$-axis, and thus the $v$-component values shown are largely negative, as indicated by colors tending from green to blue. The positive $x$-axis points in the radially outward direction, and the $z$-axis corresponds to the azimuthal direction, with component values as identified in the associated color bars. These and all subsequent displayed velocity results have been processed with an ideal, spectrally sharp filter to remove clearly spurious noise at the grid scale arising from, e.g., numerical discretization errors. The sharp cutoff scale for this filter is chosen at wavenumber 


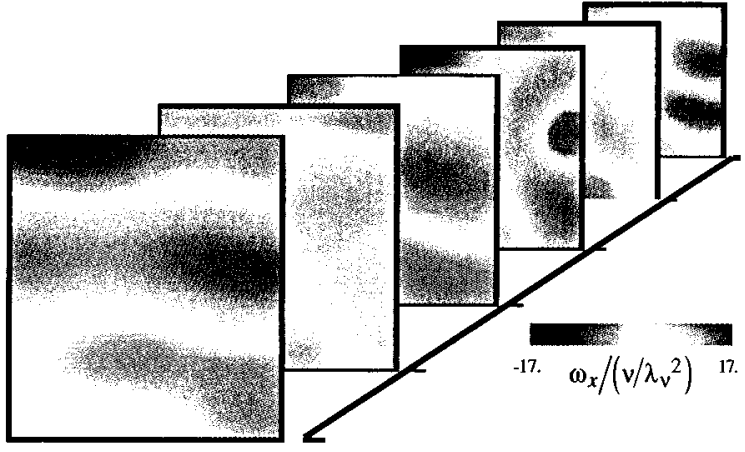

(a)

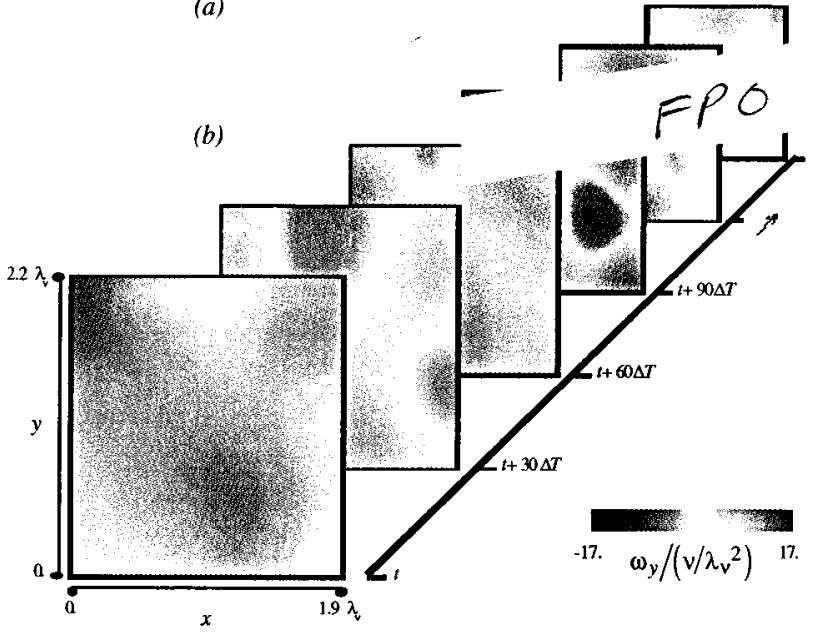

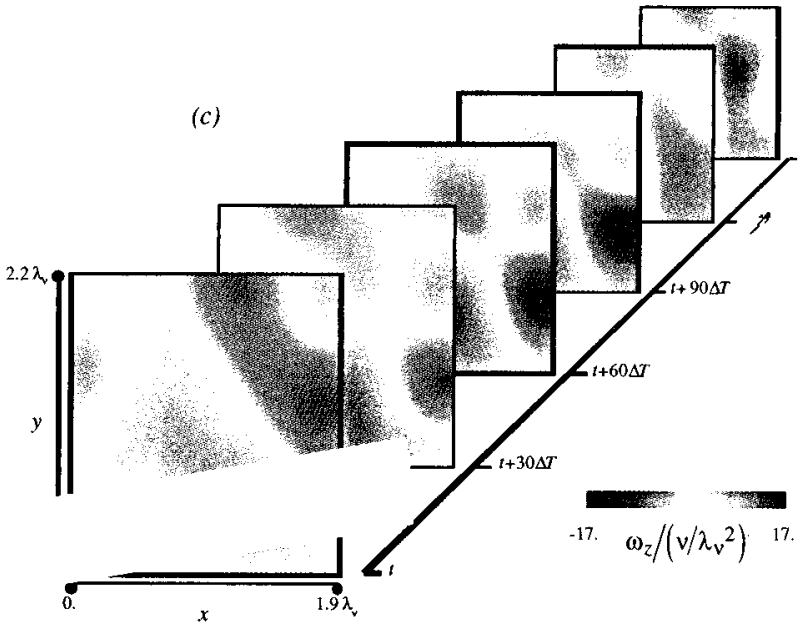

FIG. 14. The components of the vorticity vector field $\omega(\mathbf{x}, t)$, from the velocity field results of Fig. 12. Shown are (a) the $\omega_{x}(\mathbf{x}, t)$ component, (b) the $\omega_{y}(\mathbf{x}, t)$ component, and the $\omega_{z}(\mathbf{x}, t)$ component (c).

$k=2 \pi / \lambda_{\nu}$, so that the results at scales larger than the estimated finest velocity gradient length scale remain unaffected.

The resulting velocity field $\mathbf{u}(\mathbf{x}, t)$ is much smoother than the scalar field, consistent with the $\lambda_{\nu}=(2075)^{1 / 2} \cdot \lambda_{D}$ disparity in gradient length scales between these two fields. Each of the data planes spans roughly two viscous scales $\lambda_{\nu}$ in the $x$ - and $y$-directions, as seen in Fig. 9. This limited range of accessible length scales is the primary disadvantage of Sc $\gg 1$ scalar field data in scalar imaging velocimetry studies of turbulent flow structure, precluding for example the determination of spatial structure, spectra, and other information at all but very small length scales. These results nevertheless offer access to complete velocity gradient tensor information at the small scales of turbulent flows. In addition, as will be seen in Section III D 3, information on the inertial range of scales is available from these measurements through the much longer temporal dimension of these fourdimensional velocity field results.

Figure 10 presents the velocity fields of Fig. 9 in the more familiar and perhaps more intuitive form of vector projections. Shown are the projections of the $u$ - and $v$-components of the velocity field $\mathbf{u}(\mathbf{x}, t)$, and the $u$ - and $w$-components, into the $x$-y plane. The $v$-component of $\mathbf{u}(\mathbf{x}, t)$ has had the mean streamwise component subtracted, to emphasize the structure of the flow field. While such vector projections allow orientation information to be better comprehended, it will be seen in the following sections that in most of the fields considered, more insight can be gained by viewing the sample results as colormaps of the type in Fig. 9.

\section{Time series and probability distributions}

The results in Figs. 9 and 10 demonstrate the spatial information on the velocity field available from these scalar imaging velocimetry measurements with the scalar field data of Section II. Of course, these SIV measurements of the velocity and velocity gradient fields may be repeated in time as well. Figure 11 shows a time series of six scalar field data planes from the data set R0628. This figure is similar to Fig. 4 , except that the time step shown is much larger, with successive planes separated by $30 \Delta T$, where $\Delta T$ is the time separation of the scalar field data volumes. This larger temporal separation is reflective of the longer time scales on which changes in the velocity field occur. In this section, results will be presented for the velocity and velocity gradient fields in the same six planes, as well as for the statistics obtained over the roughly 300 or more time steps at which velocity results were found for each of the three cases.

\section{Results for $\mathrm{u}(\mathrm{x}, t)$ and $\nabla \mathrm{u}(\mathrm{x}, t)$}

The components of the velocity field $\mathbf{u}(\mathbf{x}, t)$ corresponding to each of the scalar field planes in Fig. 11 are shown in 


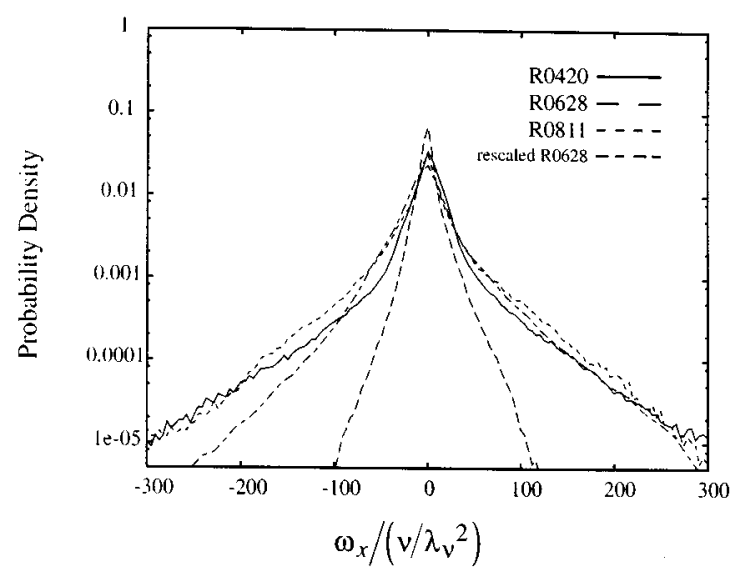

(a)

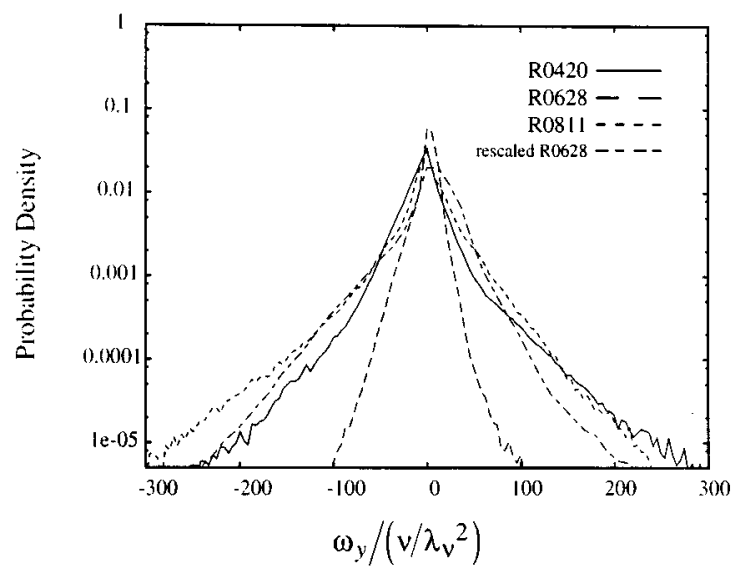

(b)

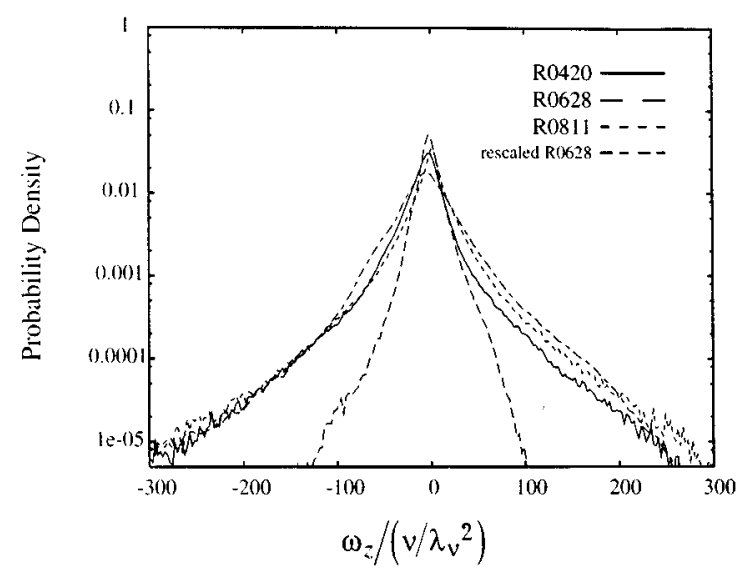

(c)

FIG. 15. Probability distributions of the components of the vorticity vector $\omega(\mathbf{x}, t)$. Shown are (a) the $\omega_{x}(\mathbf{x}, t)$, (b) the $\omega_{y}(\mathbf{x}, t)$, and (c) the $\omega_{z}(\mathbf{x}, t)$ distributions.

Fig. 12. It is evident in this figure that the temporal separation of $30 \Delta T$ between successive planes is indeed characteristic of the time scales in the velocity field. This can be seen, for example, in the $v$-component field results in Fig. 12, where there is a clear evolution over the time span shown. This is a partial validation of the results obtained by the SIV technique, since no shared scalar field information is used in extracting the velocity field in the six time steps shown. That the results nevertheless show a consistent structure, which evolves clearly over these time steps, suggests that the ve- locity fields obtained are primarily driven by the scalar transport condition $E_{1}$ and less so by the continuity and smoothness conditions $E_{2}$ and $E_{3}$.

Figure 13 presents probability density functions of the velocity component values, for each of the three data sets R0420, R0628 and R0811. The strong tendency for negative values of the $v$-component of the velocity is, of course, consistent with the negative $y$-axis being the streamwise direction. The mean streamwise velocity at the measurement location is $\sim 30 \nu / \lambda_{\nu}$. It is, however, apparent in Fig. 13 that the velocity field data for each of the three cases shown do not span sufficiently long times for the statistics to have converged to their stationary form. Although the probability distributions of the individual velocity component values are comprised of well over a million points for each of the three cases, these span a total time $\tau$ of only about two local outer time scales. Therefore $\tau \approx 2(\delta / U)$, whereas a duration of at least ten outer time scales $(\delta / U)$ is typically required to obtain converged statistics of the velocity component values. The time needed for convergence of velocity gradient statistics is, in contrast, much shorter, since these scale with the local advection time scale $\left(\lambda_{\nu} / U\right)$. In terms of this advection time scale, the data sets each span a total time $\tau \approx 75\left(\lambda_{\nu} / U\right)$, which is adequate for velocity gradient statistics to have converged to their stationary form. Indeed, as will be seen below, the velocity gradient statistics obtained from these scalar imaging velocimetry measurements are essentially converged.

The three components of the vorticity vector field $\omega(\mathbf{x}, t)$, determined directly from the velocity field results of Fig. 12 as $\nabla \times \mathbf{u}(\mathbf{x}, t)$, are shown in Fig. 14. These results represent the first non-invasive measurements of the full three-component vector vorticity field in a turbulent flow. By giving the vorticity at a very large number of closely spaced points in space and time, these measurements also allow examination of the spatial structure and temporal dynamics of the vorticity field. Probability distributions of the vorticity field component results are presented in Fig. 15. Similarly, results for the three normal and three shear components of the strain rate tensor field $\varepsilon(\mathbf{x}, t) \equiv \frac{1}{2}\left(\nabla \mathbf{u}+\nabla \mathbf{u}^{T}\right)$ obtained from these data are shown respectively in Figs. 16 and 18, while the corresponding probability distributions are given in Figs. 17 and 19. Collectively, these give all nine components of the velocity gradient tensor field $\nabla \mathbf{u}(\mathbf{x}, t)$. It can be seen from the probability distributions of Figs. 15, 17 and 19 that the results for cases R0420 and R0811, when normalized by inner variables, are substantially in agreement, and are also symmetric about zero. This suggests that the distributions obtained for these cases are converged. The scaling of the tails of the R0420 and R0811 results in these semilogarithmic plots are also significant. In the chosen axes, a straight line falloff for increasing magnitudes would indicate exponential scaling of the probability density distribution. It follows in such a case that all moments of the distribution would be convergent, which is of interest in regard to models of the small scale intermittency of turbulence. The results for cases R0420 and R0811, shown in Figs. 15, 17 and 19, indeed show evidence of such an exponential decay in the scaling of the tails. 


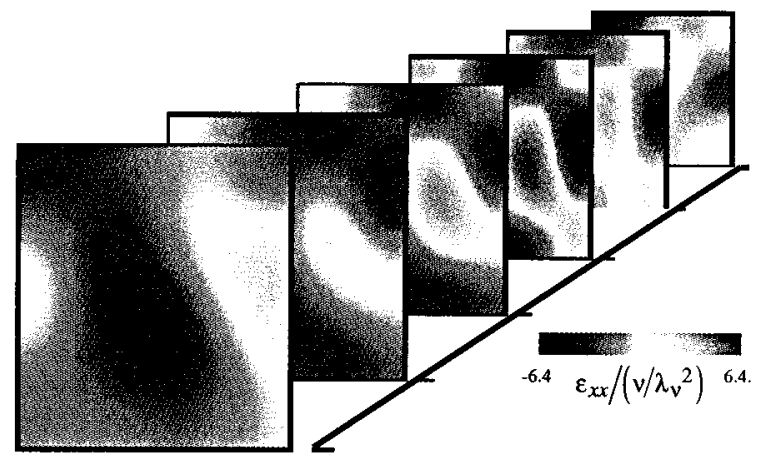

(a)
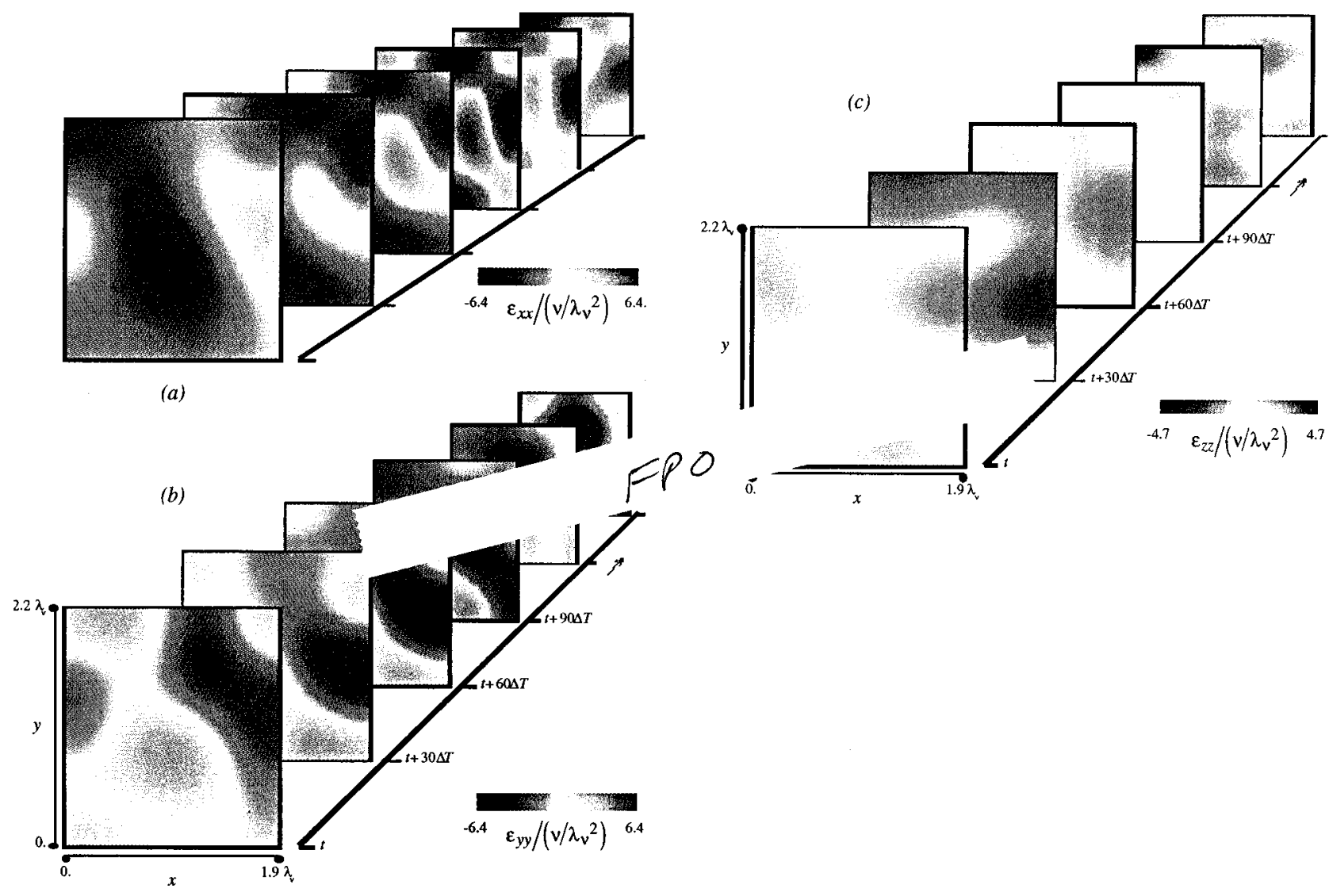

FIG. 16. The normal components of the strain rate tensor field $\varepsilon(\mathbf{x}, t)$ for the velocity field results of Fig. 12. (a) The $\varepsilon_{x x}(\mathbf{x}, t)$ results. (b) The $\varepsilon_{y y}(\mathbf{x}, t)$ results. (c) The $\varepsilon_{z z}(\mathbf{x}, t)$ results.

It should be noted that, at the finest scales, turbulence structure is widely held to be universal for high Reynolds numbers. As discussed in Refs. 3 and 4, recent direct numerical simulations of turbulent flows ${ }^{11}$ suggest that the Taylor scale Reynolds numbers at which the scalar field measurements of Section II were made are sufficiently high that the fine scale structure largely approaches its high Reynolds number form. The scaled probability distributions presented here should thus be largely similar to those obtained in other turbulent shear flows at similar and higher Reynolds numbers, when normalized by the inner reference scales. In this sense, the results reported here are believed not to be specific to the turbulent jet at these Reynolds numbers, but to be largely representative of the fine scale structure and dynamics of all high Reynolds number turbulent shear flows.

Two other aspects of these distributions are particularly striking; the differences between the R0420 and R0811 results and the results for case R0628, and the substantially smaller derivative values found in the $z$-direction for each of the sets. The latter issue was previously discussed in Section III B. Regarding the former, although the results for cases R0420 and R0811 are substantially in agreement, the R0628 case has velocity gradient component values which are uniformly lower than those for the other two cases. This suggests that the reference inner length scale $\lambda_{\nu}$ used in the normalization, which is found from the non-buoyant turbulent jet scaling laws, is smaller than the true value. The likely explanation for this is that buoyancy effects in the R0628 case were non-negligible. (Complete elimination of buoyancy effects in this flow is extremely difficult-density gradients corresponding to temperature differences of $\Delta T=0.1^{\circ} \mathrm{C}$ cause noticeable buoyancy effects, while smaller density differences can lead to buoyancy effects which are not manifested as visible differences in the growth rate of the flow. Identifying the effect of buoyancy in the latter case thus requires velocity measurements, as is done here.) The presence of buoyancy in this jet facility would act to decrease the outer scale Reynolds number at the measurement location. Such a decrease in the Reynolds number would lead to a larger value of the reference length $\lambda_{\nu}$ as well as a smaller value of the reference velocity scale $\nu / \lambda_{\nu}$, in accordance with the observations of Figs. 15, 17 and 19. Further evidence that the true velocities in the R0628 case are lower than those expected from the non-buoyant jet scaling laws can be seen in the distribution of $v$-component values in Fig. 13 . While the mean streamwise velocity (the $v$-component) for a non-buoyant jet in the R0628 case would be $-28.0 \nu / \lambda_{\nu}$, the measured $v$ values are distributed much nearer zero, with a mean value of $-6.7 \nu / \lambda_{\nu}$. This is consistent with the effect of negative buoyancy, with an accompanying decrease in the outer scale Reynolds number and a consequent larger value of $\lambda_{\nu}$. The larger $\lambda_{\nu}$ leads to longer characteristic times $\left(\lambda_{\nu} / U\right)$ and thus slower convergence of the probability distributions for this case. This also explains 


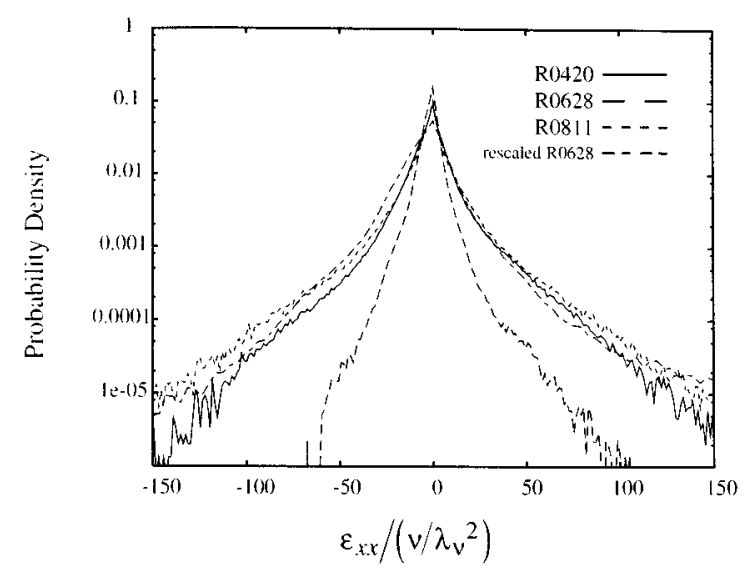

(a)

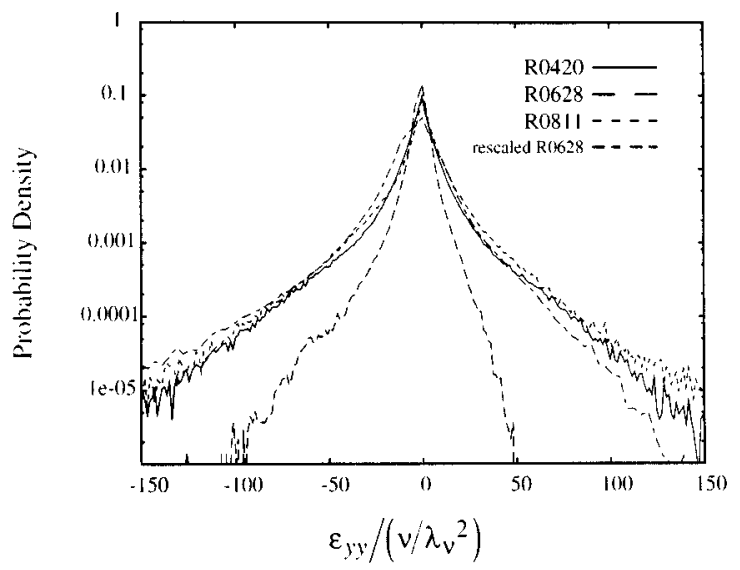

(b)

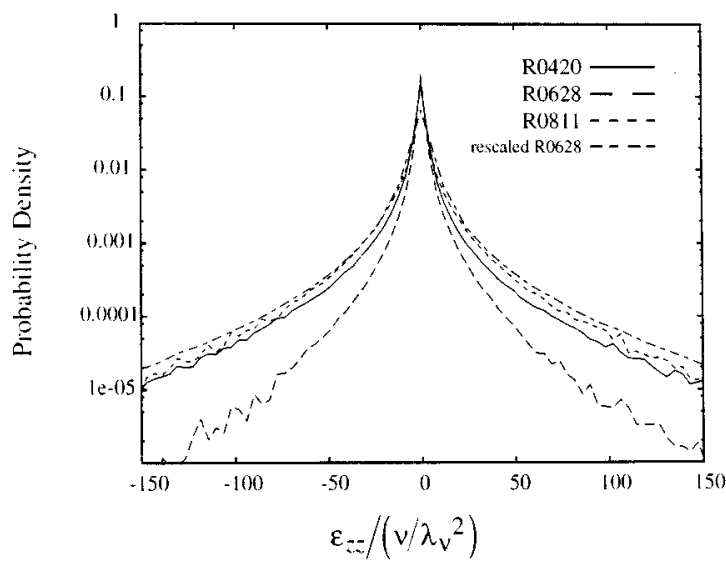

(c)

FIG. 17. Probability distributions of the normal components of the strain rate tensor $\varepsilon(\mathbf{x}, t)$. Shown are (a) the $\varepsilon_{x x}(\mathbf{x}, t)$ distribution, (b) the $\varepsilon_{y y}(\mathbf{x}, t)$ distribution, and (c) the $\varepsilon_{z z}(\mathbf{x}, t)$ distribution. The rescaled R0628 curves correspond to a different estimate of the viscous scale $\lambda_{\nu}$, as discussed in Section III D 1.

the apparent asymmetry of the probability distributions for the R0628 case, suggesting that complete statistical convergence for that case has not been achieved.

The foregoing argument suggests that the probability distributions for the R0628 case should be rescaled with an adjusted value of $\lambda_{\nu}$ which is reflective of the true viscous diffusion length scale. The adjusted value used is 1.7 times the value of $\lambda_{\nu}$ determined from the (non-buoyant) jet scaling. The dashed-dotted curves in the probability distributions of Figs. 15, 17, and 19 (as well as in all remaining distributions presented here) represent the data of the R0628 case, as normalized by this adjusted value of $\lambda_{\nu}$. Though the resulting renormalized curves obviously retain their original asymmetry, these distributions are otherwise in good agreement with the R0420 and R0811 curves. This will be seen more dramatically in Fig. 20, where the rescaling by the adjusted inner scale $\lambda_{\nu}$ produces excellent agreement of the velocity gradient probability distributions for both the buoyant and non-buoyant turbulent jet cases. This agreement supports the idea that the inner scales of these turbulent flows have substantially achieved their high Reynolds number asymptotic state, independent of both the outer scale Reynolds number and the particular turbulent shear flow in which the measurements were made.

As a further note on these velocity gradient results, the agreement with the continuity condition $\nabla \cdot \mathbf{u}=0$ was quantified by measuring the fluctuation correlation between $(\partial u / \partial x)$ and $(-\partial v / \partial y-\partial w / \partial z)$, which assumes a value of 1 when continuity is exactly satisfied. The SIV measurements yielded an $x$-divergence value of 0.73 for these three data sets. This compares favorably with the range of values, from 0.25 to 0.7 , reported in hot-wire measurements (Tsinober et al. $\left.{ }^{12}\right)$.

\section{Kinematic quantities}

The velocity gradients $\nabla \mathbf{u}(\mathbf{x}, t)$ in turbulent flows are of interest in part because of their relation to various higherorder quantities connected with the kinematics of the fluid motion. Perhaps most insightful among these are quantities associated with energy density, energy transfer and energy dissipation. The transport equation for the kinetic energy density field $k \equiv \frac{1}{2} \mathbf{u} \cdot \mathbf{u}(\mathbf{x}, t)$ is

$$
\left[\frac{\partial}{\partial t}+\mathbf{u} \cdot \nabla-\frac{1}{\operatorname{Re}} \nabla^{2}\right] k(\mathbf{x}, t)=-\mathbf{u} \cdot \nabla p+\frac{1}{\operatorname{Re}} \nabla \mathbf{u}: \nabla \mathbf{u}^{T}-\frac{2}{\operatorname{Re}} \varepsilon: \varepsilon,
$$

where the last term on the right-hand side gives the kinetic energy dissipation rate $\Phi(\mathbf{x}, t) \equiv(2 / \operatorname{Re}) \varepsilon: \varepsilon(\mathbf{x}, t)$. Also of interest in understanding the kinematics of the underlying vorticity field is the enstrophy field, defined as $\frac{1}{2} \omega \cdot \omega(\mathbf{x}, t)$. The enstrophy transport equation is

$\left[\frac{\partial}{\partial t}+\mathbf{u} \cdot \nabla-\frac{1}{\operatorname{Re}} \nabla^{2}\right] \frac{1}{2} \omega \cdot \omega(\mathbf{x}, t)=\omega \cdot \varepsilon \cdot \omega-\frac{1}{\operatorname{Re}} \nabla \omega: \nabla \omega$.

The terms on the right-hand side are respectively the enstrophy production rate and the enstrophy dissipation rate. Unlike Eq. (14), however, pressure is absent.

Figures 20 and 21 presents results for the true kinetic energy dissipation rate field $\Phi(\mathbf{x}, t)$, the enstrophy field $\frac{1}{2} \omega \cdot \omega(\mathbf{x}, t)$ and the enstrophy production rate field $\omega \cdot \varepsilon \cdot \omega(\mathbf{x}, t)$. These are found from the velocity field results of Fig. 12, and represent the first, direct, non-intrusive measurements of these kinematic quantities in turbulent flows. It is notable that the energy dissipation rate field and the enstrophy field in Fig. 21 are both relatively "spotty," with large values occurring very rarely. Such high internal intermittency in these fields has been recognized since the origi- 


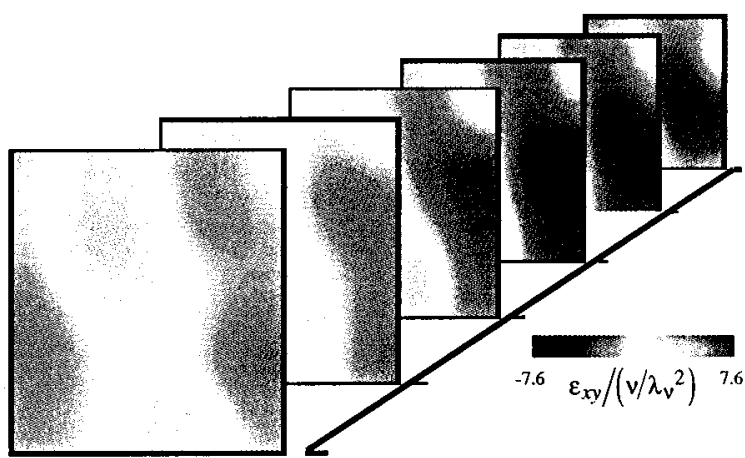

(a)

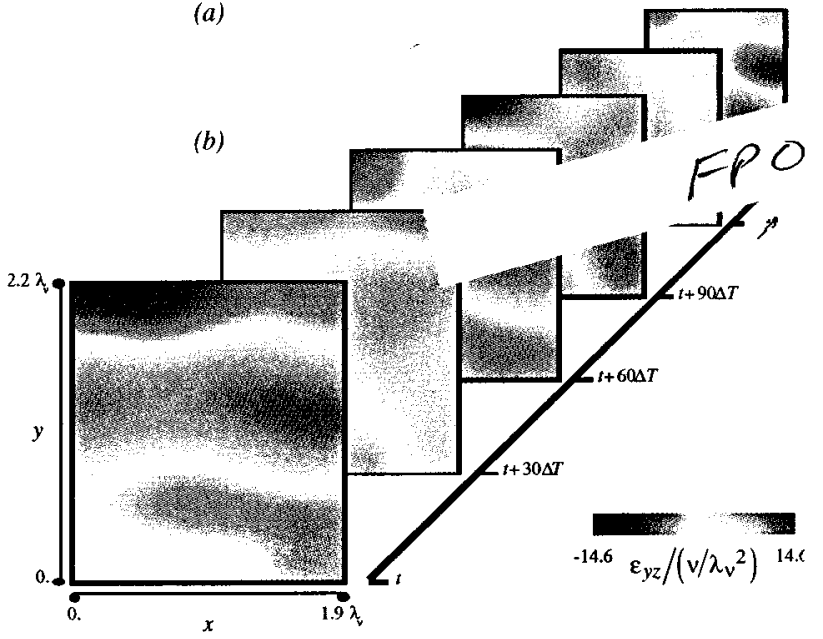

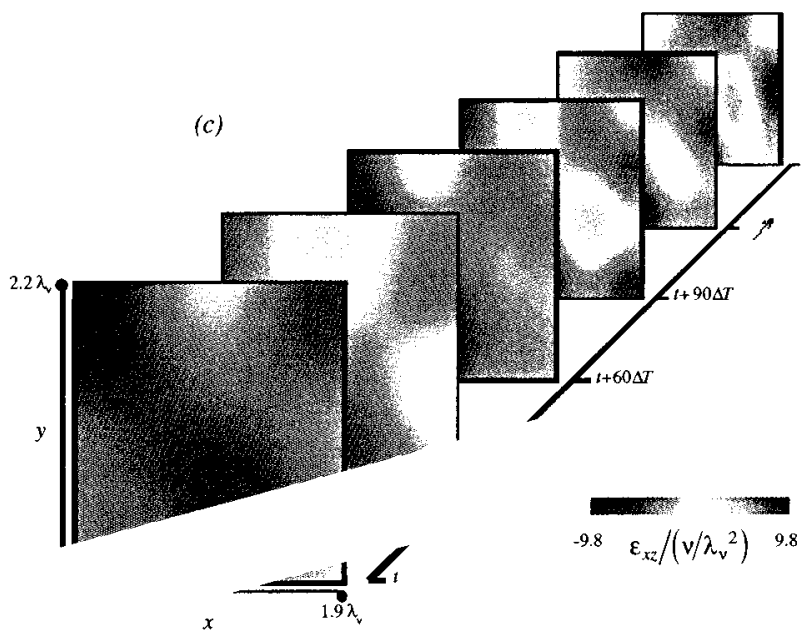

FIG. 18. The shear components of the strain rate tensor field $\varepsilon(\mathbf{x}, t)$ for the velocity field results of Fig. 12. (a) The $\varepsilon_{x y}(\mathbf{x}, t)$ results, (b) the $\varepsilon_{y z}(\mathbf{x}, t)$ results, and the $\varepsilon_{x z}(\mathbf{x}, t)$ results $(\mathrm{c})$.

nal indirect measurements of Batchelor and Townsend, ${ }^{13}$ and forms the basis for most higher-order dynamical models of the small scales of turbulence. Both of these fields are second-order in the velocity gradient components; in contrast, the enstrophy production rate field in Fig. 21 is thirdorder in the velocity gradients, and thus is even more highly intermittent. This can be seen directly in the probability distributions of these quantities for the three cases considered, given in Fig. 20. The extreme rarity of large magnitudes in all of these quantities is readily apparent. It can also be seen that the results for all three cases collapse to an essentially universal form when the $\lambda_{\nu}$ value for the R0628 case is rescaled to account for the presumed buoyancy effects. Though the outer scale Reynolds numbers for the three cases considered do not differ greatly, the agreement of the buoyant and non-buoyant jet results, when normalized by appropriate inner variables, suggests that these results are largely indicative of the high Reynolds number form of these distributions. (Further supporting evidence for this will be seen in the following section when inertial range structure function exponents, obtained from the kinetic energy dissipation rate distributions in Fig. 20, are compared with previous results of Anselmet et al. ${ }^{14}$ at high Reynolds numbers.)

To account for the internal intermittency of turbulence, Kolmogorov ${ }^{15}$ postulated a log-normal distribution for the energy dissipation. While this has been shown to be strictly inconsistent with the physics of incompressible flow, as discussed for example by Frisch, ${ }^{16}$ gross departures from the log-normal dissipation model should be manifested primarily in high-order statistical quantities. Figure 22 compares the distribution of kinetic energy dissipation rates for the R0811 case with a log-normal distribution, which is Gaussian in the displayed axes. Also shown is a comparison of the distribution of enstrophy field values for this case with a log-normal distribution. In both fields, the log-normal approximation gives a relatively good model of the distributions for all but the lowest values. In particular, it appears that the log-normal model underestimates the occurrence of low values in these fields. Departures from strict log-normal scaling will become clear in examining the inertial range structure function exponents in the following section.

To understand the results for the enstrophy production rate $\omega \cdot \varepsilon \cdot \omega=\omega_{i} \varepsilon_{i j} \omega_{j}$ in Figs. 20 and 21, it is helpful to write this in a more physically intuitive form as

$$
\omega_{i} \varepsilon_{i j} \omega_{j}=\varepsilon_{\omega \omega}(\omega \cdot \omega),
$$

where $\varepsilon_{\omega \omega}$ is the normal strain rate in the direction of the vorticity $\omega$. Alignment of the vorticity vector with a positive strain rate axis $\left(\varepsilon_{\omega \omega}>0\right)$ therefore results in the production of enstrophy, while alignment with a compressional strain axis decreases the enstrophy. The vorticity-strain rate alignment is discussed in some detail in Section III D 5. We ob- 


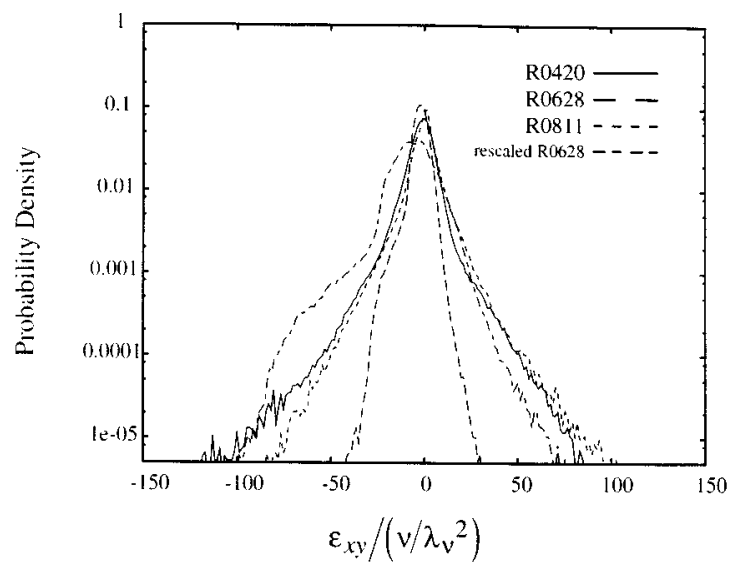

(a)

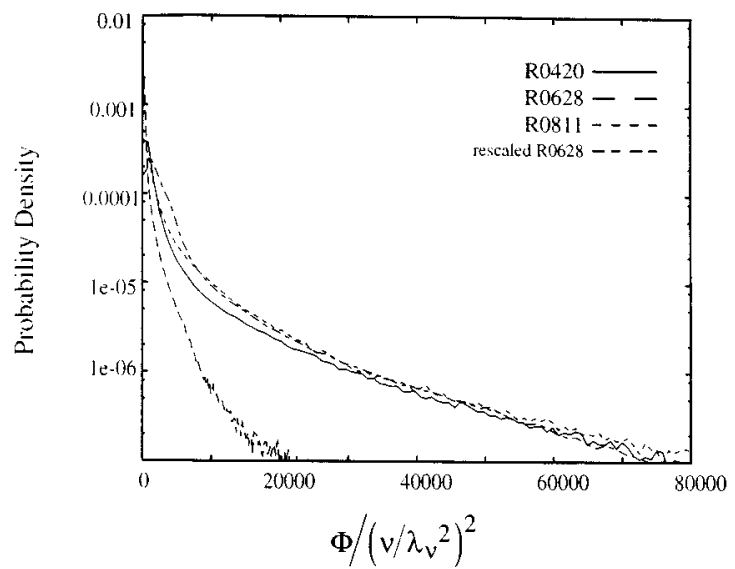

(a)
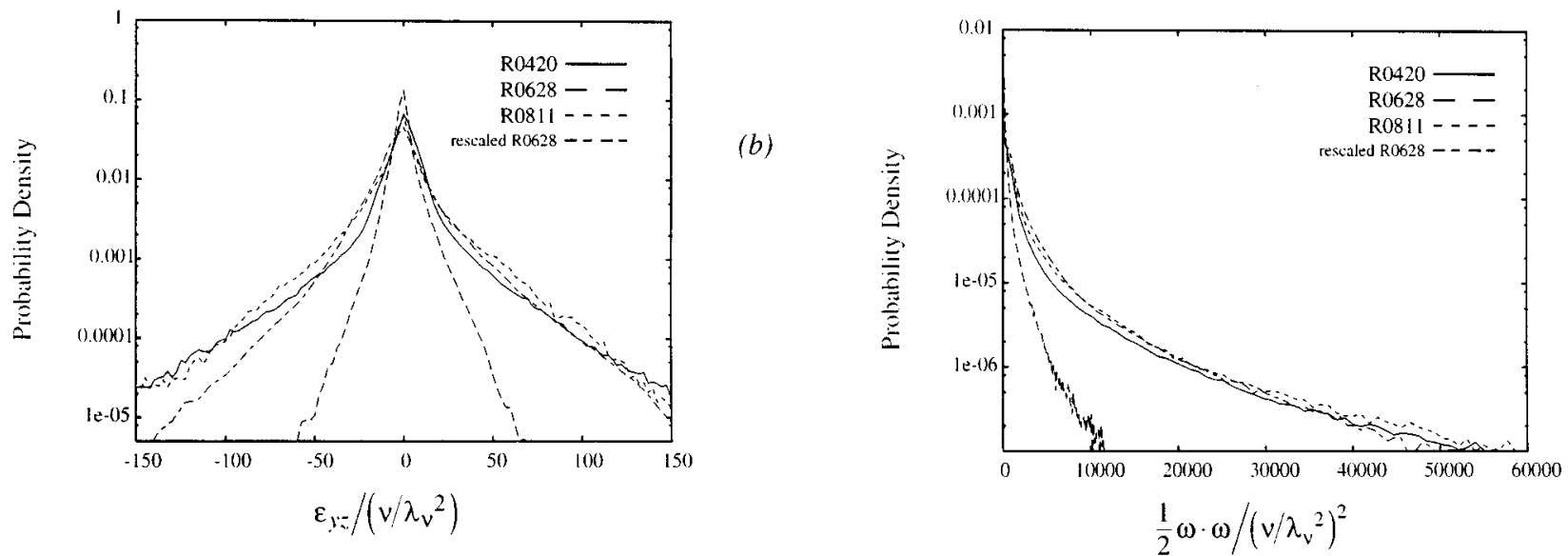

(b)
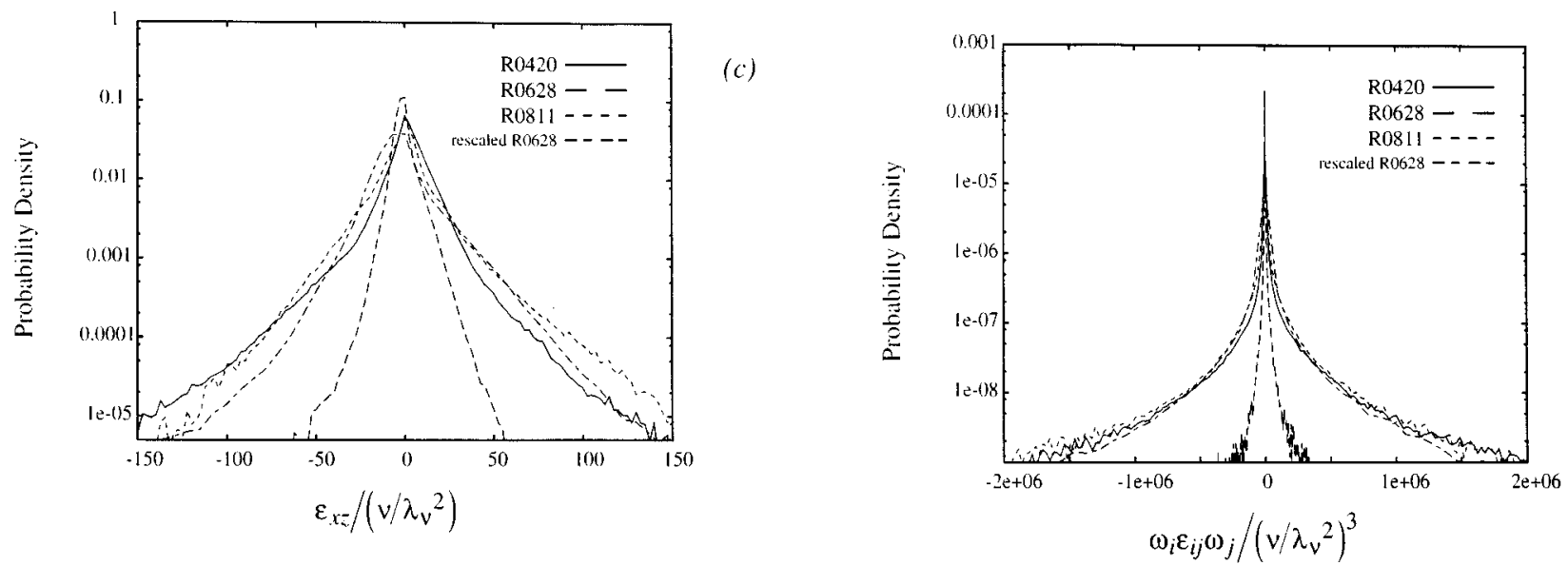

(c)

FIG. 19. Distributions of the shear components of the strain rate tensor $\varepsilon(\mathbf{x}, t)$. Shown are (a) the distribution of $\varepsilon_{x y}(\mathbf{x}, t)$ (b) the $\varepsilon_{y z}(\mathbf{x}, t)$ distribution, and the $\varepsilon_{x z}(\mathbf{x}, t)$ distribution (c).

serve here that for each of the data sets R0420, R0628 and R0811 the enstrophy production has a positive mean, suggesting that the intensification of enstrophy by stretching occurs more frequently than its diminution by compression, in agreement with the hot-wire experiments of Tsinober et al. ${ }^{12}$ and the computations reported by Rogers and Moin. ${ }^{17}$

\section{Inertial range scaling exponents}

Though the spatial extent of the present measurements is limited to about two inner scales $\lambda_{\nu}$, the temporal extent

FIG. 20. Probability distributions for (a) the kinetic energy dissipation $\Phi(\mathbf{x}, t) \equiv 2 \varepsilon: \varepsilon / \operatorname{Re}$, (b) the enstrophy $\omega \cdot \omega / 2$, and (c) the enstrophy production $\omega \cdot \varepsilon \cdot \omega=\omega_{i} \varepsilon_{i j} \omega_{j}$.

typically spans over 75 advection time scales $\left(\lambda_{\nu} / U\right)$. As a result, the accessible spatial structure in the velocity and velocity gradient fields is restricted to length scales well within the dissipation range. However, the accessible temporal scales extend well into the inertial range, and make possible the examination of the scaling properties of turbulence fields well into this range. This may be done equivalently in either the spectral or physical domains. In the physical domain, the scaling can be related to the moments of the distribution of 


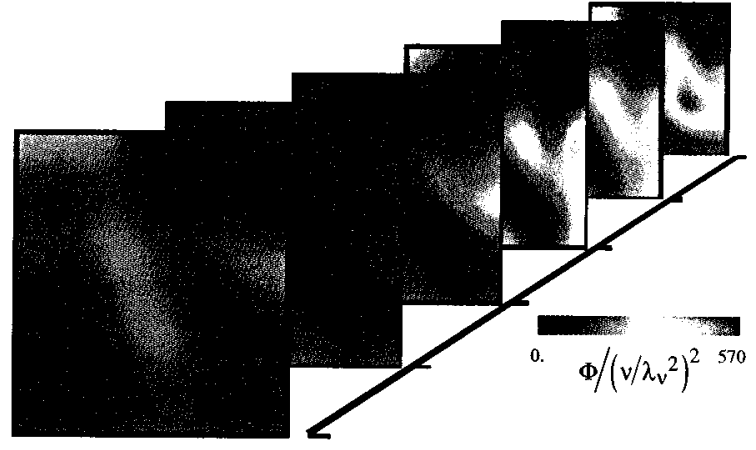

(a)

(b)
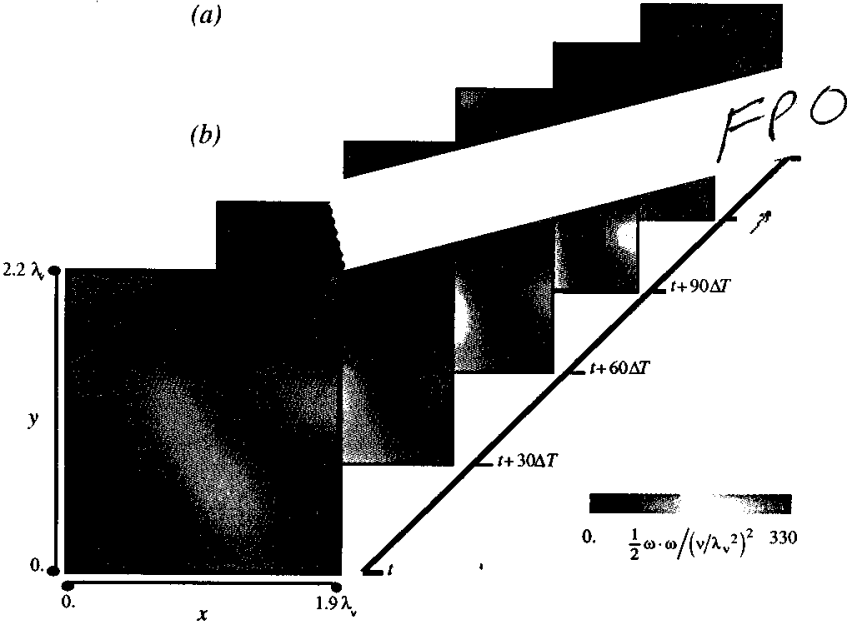
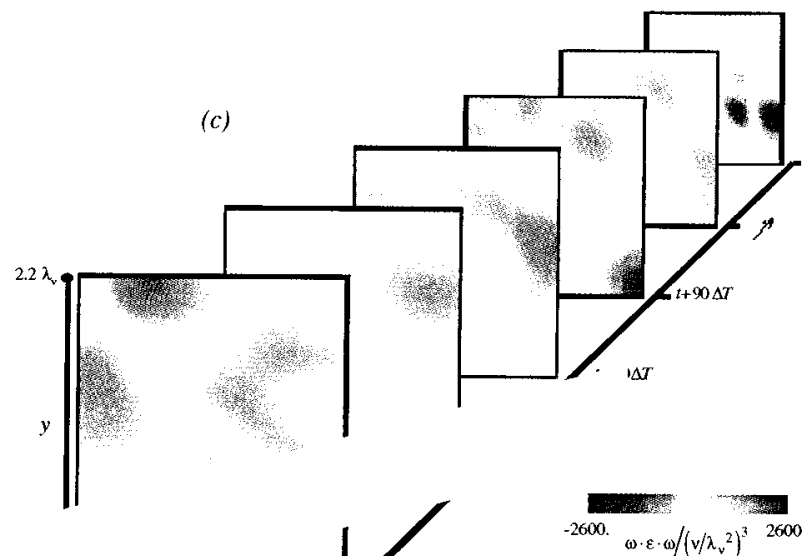

FIG. 21. Key kinematic quantities formed from the velocity gradient field results of Figs. 14-18. (a) The kinetic energy dissipation term $\Phi(\mathbf{x}, t) \equiv 2 \varepsilon: \varepsilon / \operatorname{Re}$, (b) the enstrophy $\omega \cdot \omega / 2$, and (c) the enstrophy production term $\omega \cdot \varepsilon \cdot \omega=\omega_{i} \varepsilon_{i j} \omega_{j}$.

the kinetic energy dissipation rate field values $\Phi(\mathbf{x}, t)$. This is generally done in terms of the two-point structure functions, which scale in the inertial range as

$$
\left\langle(\Delta u)^{q}\right\rangle \propto L^{q / 3}\langle\Phi\rangle^{q / 3}\left(\frac{r}{L}\right)^{\zeta_{q}}
$$

The inertial range scaling exponents $\zeta_{q}$ are given by

$$
\zeta_{q}=\frac{1}{3} q-\mu_{q},
$$

where the $\mu_{q}$ are the intermittency corrections to the inertial Kolmogorov ${ }^{18}$ predictions $\zeta_{q}=\frac{1}{3} q$. These corrections are related to the moments of $\Phi(\mathbf{x}, t)$ in Figs. 20 and 22 as

$$
\mu_{q} \equiv \mu_{6} \frac{\log \left(\left\langle\Phi^{q / 3}\right\rangle /\langle\Phi\rangle^{q / 3}\right.}{\log \left(\left\langle\Phi^{2}\right\rangle /\langle\Phi\rangle^{2}\right.},
$$

where the only free parameter is $\mu_{6}$.

Figure 23 presents the structure function exponents $\zeta_{q}$ for $2 \leqslant q \leqslant 16$ determined from the $\Phi$ distribution in Fig. 22 . Also shown are the curves giving the exponents for the original Kolmogorov 1941 (uniform) and 1962 (log-normal) predictions. The plotted symbols are the exponents found by Anselmet et al. ${ }^{14}$ from two-point measurements of the structure functions in a turbulent duct at $\operatorname{Re}_{\lambda_{T}}=515$ and in a turbulent jet at $\operatorname{Re}_{\lambda_{T}}=536$ and 852. The log-normal curve shown uses a value of $\mu_{6}=0.2$, while the two curves corre- sponding to the present R0811 measurements of $\Phi(\mathbf{x}, t)$ use values of 0.2 and 0.25 , in accordance with the estimate of $\mu_{6}=0.2 \pm 0.05$ obtained by Anselmet et al.

There are several observations of particular interest in the results shown in Fig. 23. The good agreement of the SIV results for the inertial range structure function exponents for $\mu_{6}=0.25$ with the measurements of Anselmet et al. ${ }^{14}$ at least up to $q \approx 14$, supports the conclusion that the probability distributions obtained from the SIV measurements are largely indicative of their high Reynolds number asymptotic forms. In this regard it is noteworthy both that the results of Anselmet et al. were obtained at higher Taylor scale Reynolds numbers than were the present measurements, and include measurements obtained in a fundamentally different turbulent flow; this agreement between the SIV results and those of Anselmet et al. for both the exponents $\zeta_{q}$ and the value of $\mu_{6}$ comes despite the high susceptibility of the higher-order structure function exponents to errors in the underlying velocimetry measurements. Second, the close agreement of the present results for $q=2$ with the Kolmogorov result $\zeta_{2}=2 / 3$ (the Kolmogorov 2/3-law) implies a $k^{-5 / 3}$ scaling in the kinetic energy spectrum $E(k)$ with wave number $k$ (the corresponding Kolmogorov -5/3-law). Finally, although Fig. 22 indicated a roughly log-normal form for the distribution of true kinetic energy dissipation rates, the 


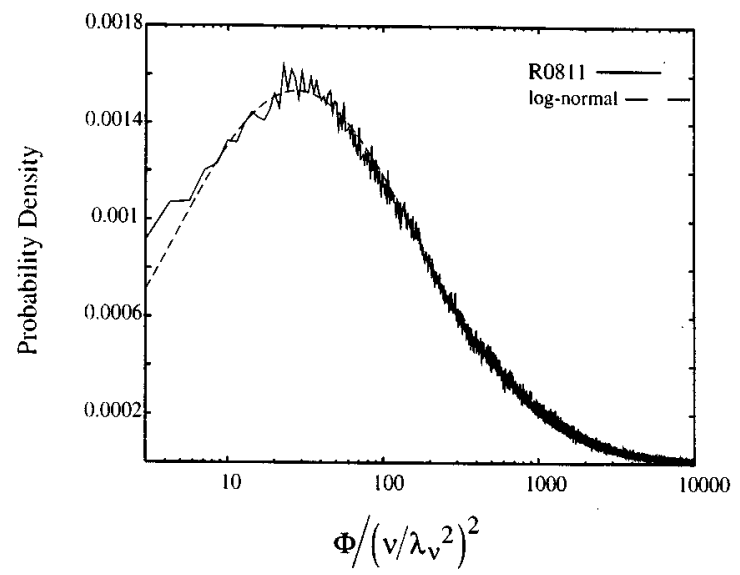

(a)

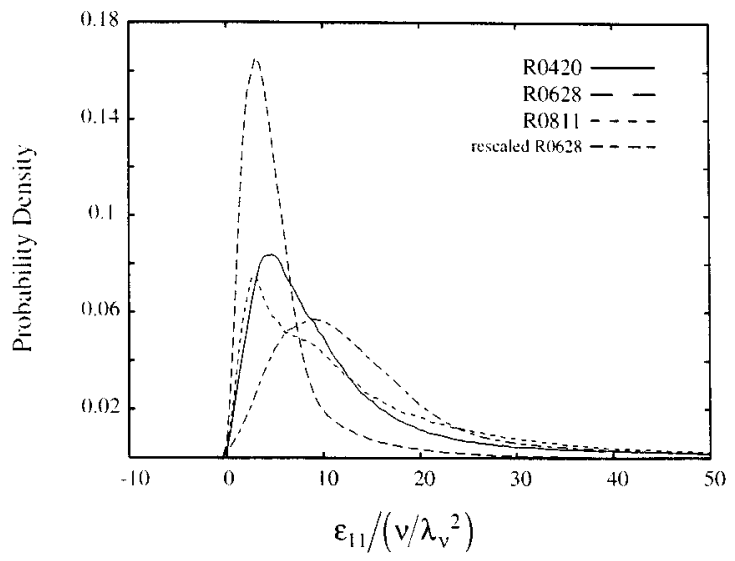

(a)
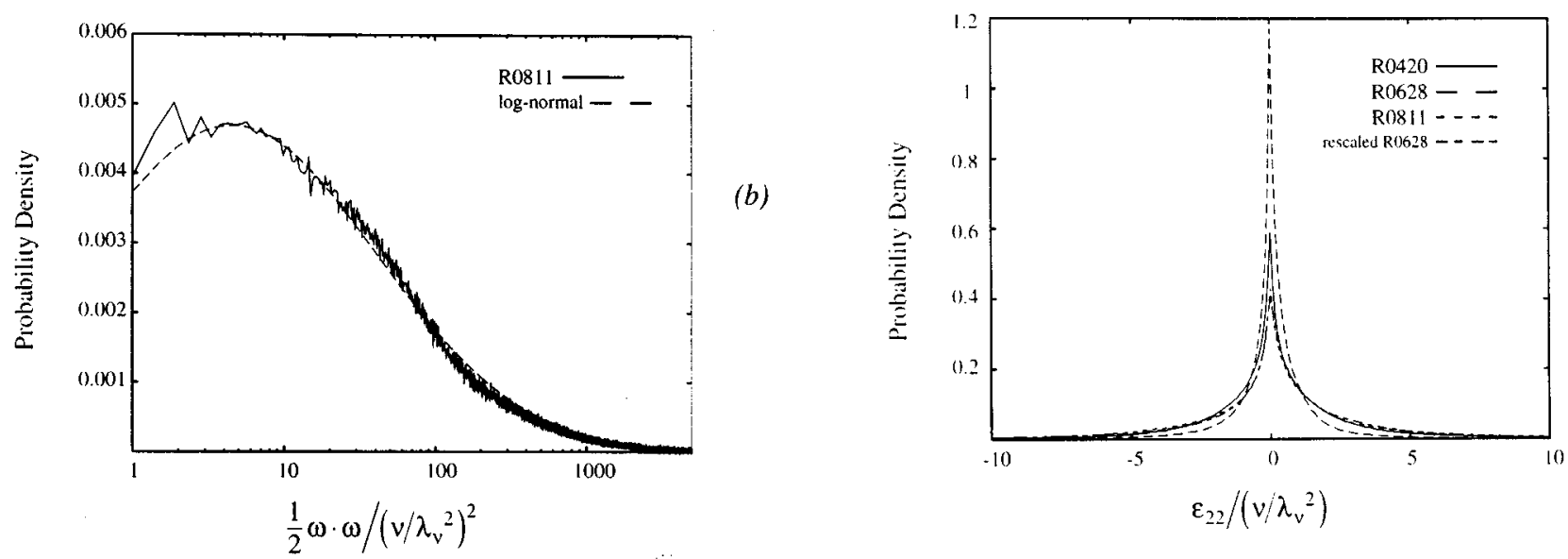

(b)

(b)

FIG. 22. Distributions of (a) the kinetic energy dissipation rate $\Phi(\mathbf{x}, t)$ and (b) the enstrophy $\omega \cdot \omega(\mathbf{x}, t) / 2$ for the R0811 case, compared with lognormal distributions.

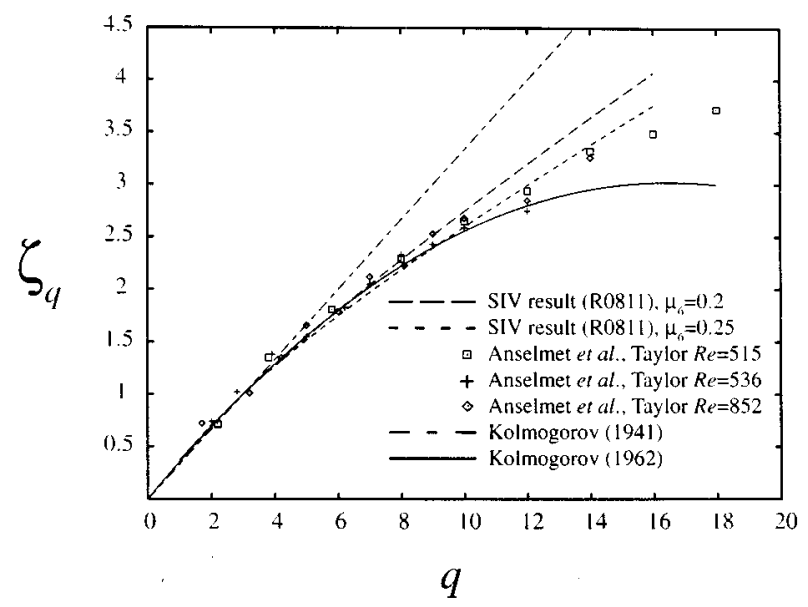

FIG. 23. Structure function exponents $\zeta_{q}$ for inertial range scalings. The log-normal model represents the original intermittency correction of Kolmogorov (Ref. 15). The symbols represent the results of Anselmet et al. (Ref. 14), while the R0811 curves represent the $\zeta_{q}$ estimated from the R0811 measurements of $\Phi(\mathbf{x}, t)$ (Fig. 22). The dashed-dotted line is the original theory of Kolmogorov (Ref. 18), uncorrected for intermittency effects.

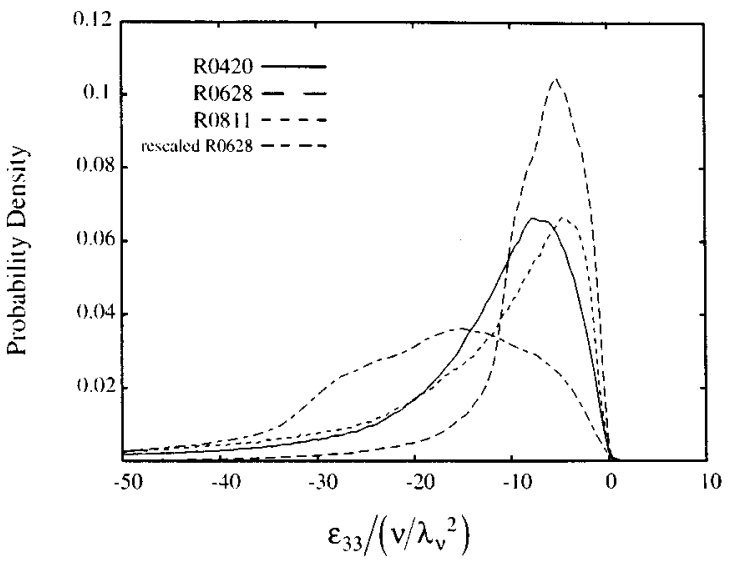

(c)

FIG. 24. Distributions of the principal strain rate eigenvalues. Shown are the results for (a) the most extensional principal strain rate $\varepsilon_{11}$, (b) the intermediate principal strain rate $\varepsilon_{22}$, and (c) the most compressive principal strain rate $\varepsilon_{33}$.

present results for the corresponding structure function exponents show clear departures from strict log-normal scaling.

\section{Structure of the strain rate field}

The strain rate field $\varepsilon(\mathbf{x}, t)$ is key to the physical structure which produces the small scale intermittency found in the kinetic energy dissipation, enstrophy and other velocity gradient fields in turbulent flows. The symmetry of the strain rate tensor $\varepsilon \equiv \frac{1}{2}\left(\nabla \mathbf{u}+\nabla \mathbf{u}^{T}\right)$ allows it to be written in terms of 


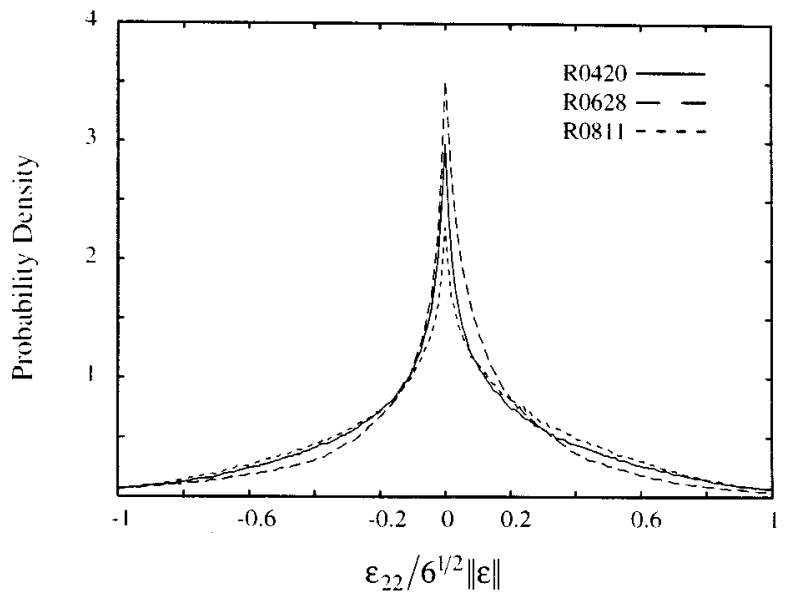

FIG. 25. Distributions of the intermediate strain rate eigenvalue $\varepsilon_{22}$, normalized to lie in the range $[-1,1]$. These curves show a positive bias for the intermediate strain rate, corresponding to the topology of two extensional and one compressive strain rates. There is at the same time a notable tendency towards the two-dimensional flow case of $\varepsilon_{22}=0$, as discussed in Sections III D 1 and III D 4.

orthogonal principal axes, with principal strain rates $\varepsilon_{11} \geqslant \varepsilon_{22} \geqslant \varepsilon_{33}$ and associated eigenvectors $\hat{e}_{\varepsilon_{11}}, \hat{e}_{\varepsilon_{22}}$ and $\hat{e}_{\varepsilon_{33}}$. Continuity requires

$$
\varepsilon_{11}+\varepsilon_{22}+\varepsilon_{33}=0,
$$

so $\varepsilon_{11} \geqslant 0$ and $\varepsilon_{33} \leqslant 0$. The structure of the local strain rate field can thus be characterized by a single parameter $\sigma \equiv \varepsilon_{22} / \varepsilon_{11}$, as

$$
\varepsilon=\left(\varepsilon_{11}, \varepsilon_{22}, \varepsilon_{33}\right)=\varepsilon_{11}(1, \sigma,-(1+\sigma)),
$$

where $\sigma$ is constrained by the continuity condition to $-1 / 2 \leqslant \sigma \leqslant 1$. There are two possible topologies for the local strain rate field, which depend upon the sign of $\sigma$. Since $\varepsilon_{11} \geqslant 0$, the sign of $\sigma$ is simply the sign of the intermediate principal strain $\varepsilon_{22}$. When $\sigma>0$ there are two extensional

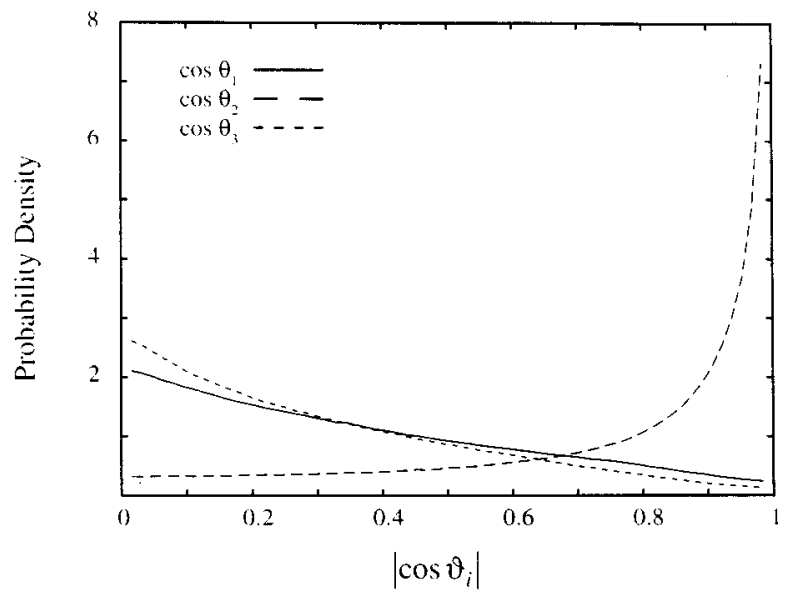

FIG. 26. Alignment of the vorticity vector $\omega$ with the principal axes of the strain rate tensor $\varepsilon$, expressed in terms of the cosine of the angles $\theta_{i}$ between $\omega$ and the principal strain rate eigenvectors $\hat{e}_{\varepsilon_{i i}}$. The tendency for the vorticity vector $\omega$ to align with the intermediate strain rate eigenvector $\hat{e}_{\varepsilon_{22}}$, while being directed away from $\hat{e}_{\varepsilon_{11}}$ and $\hat{e}_{\varepsilon_{33}}$, is very evident.

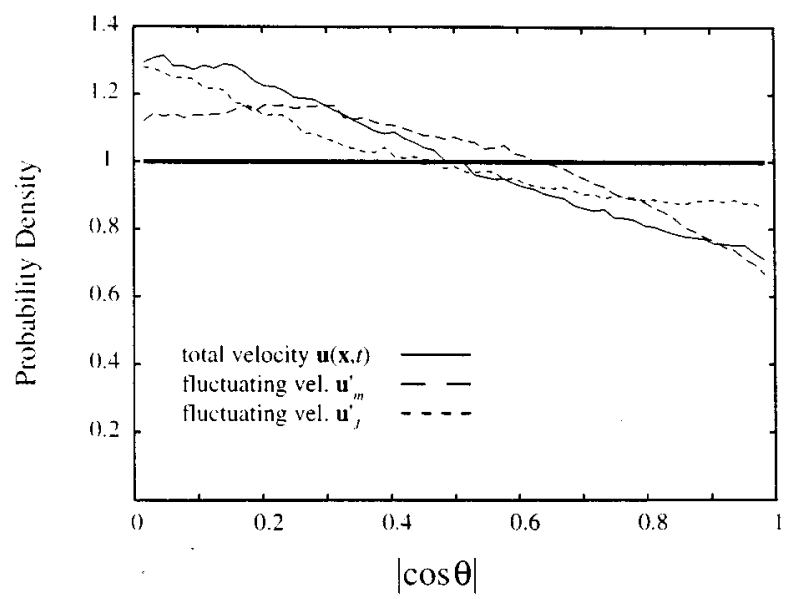

FIG. 27. Probability distributions of the relative helicity density $h(\mathbf{x}, t)=\mathbf{u} \cdot \omega /(|\mathbf{u} \| \omega|)$ for the R0420 and R0811 results. Shown are the values of $|h|$ when defined for the total velocity $\mathbf{u}$, as well as for the fluctuating velocities $\mathbf{u}_{m}^{\prime}$ and $\mathbf{u}_{J}^{\prime}$ formed using the measured and expected mean velocity components, respectively. A tendency away from alignment of $\mathbf{u}$ and $\omega$, and thus away from helical behavior, is evident.

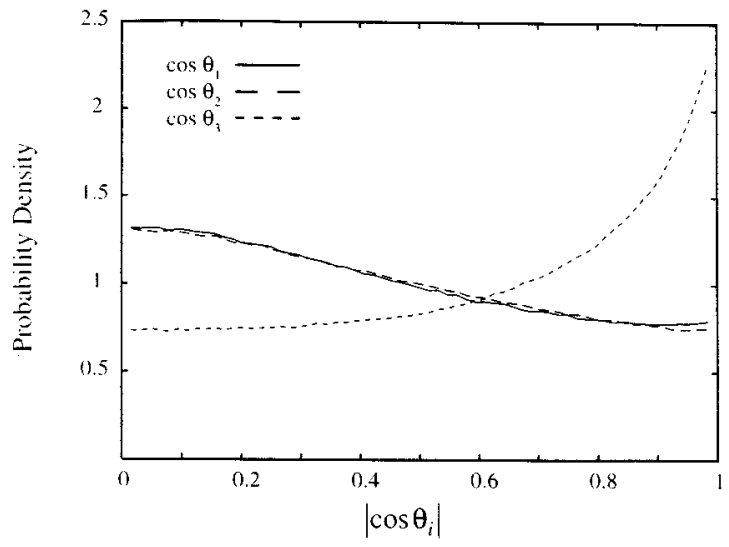

(a)

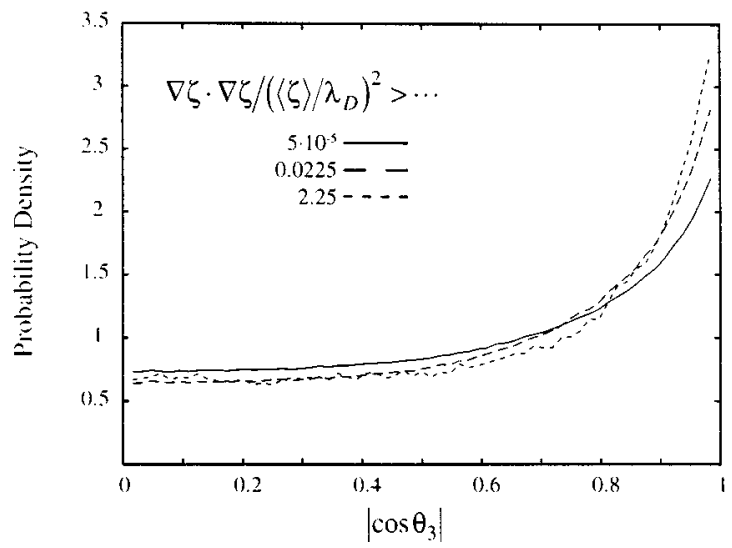

(b)

FIG. 28. Alignment of the scalar gradient vector $\nabla \zeta$ with the principal axes of the strain rate tensor. The angles between $\nabla \zeta$ and the most extensional, intermediate, and most compressional strain rate axes are denoted by $\theta_{1}$, $\theta_{2}$ and $\theta_{3}$, respectively. Shown are (a) the probability distributions for the cosines of $\theta_{1}, \theta_{2}$ and $\theta_{3}$, showing clearly the preferred alignment of $\nabla \zeta$ with the most compressive strain axis. This alignment is more pronounced at higher scalar energy dissipation rates $\nabla \zeta \cdot \nabla \zeta$, as seen in (b), where the distributions of $\cos \theta_{3}$ are shown for those points where $\nabla \zeta \cdot \nabla \zeta$ exceeds certain threshold values. 
and one compressional principal strain axes, while the $\sigma<0$ case involves one extensional and two compressional principal strain axes.

Figure 24 gives the probability distributions of the strain rate eigenvalues $\varepsilon_{11}, \varepsilon_{22}$ and $\varepsilon_{33}$. Figure 25 also shows the intermediate strain $\varepsilon_{22}$ normalized by $6^{1 / 2} \cdot\|\varepsilon\|$, where $\|\varepsilon\|=\left(\varepsilon_{11}{ }^{2}+\varepsilon_{22}{ }^{2}+\varepsilon_{33}{ }^{2}\right)^{1 / 2}$; in this form $\varepsilon_{22}$ has extreme values of -1 and 1 . This plot may be directly compared with similar plots in Ashurst et al., ${ }^{19}$ She et al. ${ }^{20}$ and Tsinober et $a{ }^{12}{ }^{12}$ In similar fashion to those results, Fig. 25 shows a tendency towards positive values of $\varepsilon_{22}$, with $\varepsilon_{22}>0$ for $51 \%, 54 \%$ and $53 \%$ of points in the R0420, R0628 and R0811 cases respectively. These values are, however, lower than the figure of $\sim 65 \%$ quoted in Tsinober et al. ${ }^{12}$ This lessened tendency towards positive intermediate strain rate would be consistent with a lower normalized rate of strain, as discussed by Ashurst et al. ${ }^{19}$ and She et al. ${ }^{20}$ Finally, Fig. 25 shows a very marked peak in each curve near $\varepsilon_{22}=0$, corresponding to a locally two-dimensional strain field, though this is likely accentuated by the smaller $z$-derivative components discussed in Section III D 1.

\section{Vorticity vector orientation}

The strong tendency of the vorticity vector field $\omega(\mathbf{x}, t)$ in turbulent flows to align with the axis of the intermediate rate of strain $\varepsilon_{22}$ was first observed by Ashurst et al. ${ }^{19}$ using data provided by direct numerical simulations of homogeneous isotropic and sheared turbulence. Tsinober et al. ${ }^{12}$ subsequently observed the same tendency experimentally, through hot-wire measurements in turbulent grid flow and in the outer region of a flat plate boundary layer. A model based on the Euler equations that describes the dynamics of this strain-vorticity alignment is given by She et al. ${ }^{20}$.

Figure 26 shows results obtained from the present measurements for the alignment of the vorticity vector $\omega$ with the strain rate eigenvectors $\hat{e}_{\varepsilon_{i i}}$. The preference for alignment of $\omega$ with the intermediate principal strain rate axis $\hat{e}_{\varepsilon_{22}}$ is very clear. The figure also shows that $\omega$ tends away from alignment with the most extensional and compressional strain rate directions $\hat{e}_{\varepsilon_{11}}$ and $\hat{e}_{\varepsilon_{33}}$. While this contrasts with the results of Tsinober et al., ${ }^{12}$ which display essentially no correlation between the most extensional strain axis and the vorticity, the present result agrees in principle with the model of She et al. ${ }^{20}$ which describes a rotation of the most extensional axis away from the vorticity vector $\omega$. The propensity for alignment of the vorticity vector with the intermediate principal strain rate eigenvector $\hat{e}_{\varepsilon_{22}}$, and the positive bias of the associated eigenvalue $\varepsilon_{22}$ (discussed in Section III D 4 above), provide an explanation for the observed positivity of the enstrophy production rate term (Section III D 2).

The alignment between the velocity and vorticity vectors in turbulent flows, quantified by the helicity density $\mathbf{u} \cdot \omega$, has in recent years been the subject of numerous investigations. Interest has generally been focused on the relative helicity density, defined as $h(\mathbf{x}, t)=\mathbf{u} \cdot \omega /(|\mathbf{u}||\omega|)$ and giving the cosine of the angle between the vorticity vector and the velocity vector. As pointed out by Rogers and Moin, ${ }^{21}$ rela- tive helicity density is not Galilean invariant, and thus depends on the choice of the velocity. In general, either the total velocity $\mathbf{u}$ or the fluctuating velocity $\mathbf{u}^{\prime}$ are used. Figure 27 presents measured probability distributions of the absolute value of the relative helicity density field $h(\mathbf{x}, t)$ for the combined results of R0420 and R0811. Shown are the distributions of $|h|$ for three different definitions of the velocity, namely the total velocity $\mathbf{u}$, the fluctuating velocity $\mathbf{u}_{m}^{\prime}$ defined using the mean velocity components for each case found from the distributions of Fig. 13, and the fluctuating velocity $\mathbf{u}_{J}^{\prime}$ defined using the mean values from the turbulent jet scaling laws for each case. The constant distribution shown is that which would be characteristic of two uncorrelated, isotropic velocity and vorticity vector fields.

The distributions of $|h|$ in Fig. 27 indicate that, irrespective of the choice of the velocity, the velocity and vorticity vector orientations are largely uncorrelated, with a slight tendency away from helical behavior. This is manifested in the somewhat larger values of the probability density as $|h| \rightarrow 0$, and smaller values as $|h| \rightarrow 1$. While the distribution of $|h|$ obtained using the total velocity $\mathbf{u}$ may be misleading, owing to the streamwise bias of $\mathbf{u}$ and the consequent anisotropy, the distributions of $|h|$ using the two estimates $\mathbf{u}_{m}^{\prime}$ and $\mathbf{u}_{J}^{\prime}$ of the fluctuating velocity show no evidence that the true fluctuating velocity field will display notable helical properties. In fact, from Fig. 27 it seems likely that the true $\mathbf{u}^{\prime}$ will be biased away from helical behavior. This result is in agreement both with the findings of Rogers and Moin ${ }^{21}$ for the relative helicity density in simulated homogeneous turbulence with mean strain, and also with the conclusions of Wallace et al. $^{22}$ from hot-wire measurements in grid turbulence and turbulent boundary and mixing layers.

\section{Scalar gradient vector orientations}

As with the vorticity vector $\omega$ (Section III D 5), the scalar gradient vector $\nabla \zeta(\mathbf{x}, t)$ shows a preferential orientation relative to the strain rate eigenvectors. This orientation, however, differs from that shown by the vorticity vector, owing to the differing nature of the stretching term in the respective transport equations. In the vorticity transport equation, the stretching term increases the vorticity magnitude when the normal strain rate along the vorticity vector direction is positive, as can be seen in Eqs. (15) and (16). In contrast, in the scalar gradient transport equation (e.g., Buch and Dahm ${ }^{6,23}$ ) the stretching term acts to decrease the scalar gradient magnitude when the normal strain rate along the gradient vector direction is extensional (positive), and increases the gradient when the normal strain rate along the gradient vector direction is compressional. It is thus expected that the scalar gradient vector field should demonstrate a preferred local alignment with the most compressional principal axis of the strain rate tensor. This preferred alignment has been confirmed through direct numerical simulations (DNS) of passive scalar mixing in turbulence under simplified conditions.

Because the scalar imaging velocimetry technique uses measurements of the scalar field to find the underlying velocity field, the simultaneous scalar and velocity gradient fields needed to assess the alignment of $\nabla \zeta(\mathbf{x}, t)$ with the eigenvectors of $\varepsilon(\mathbf{x}, t)$ are available to the present study. 


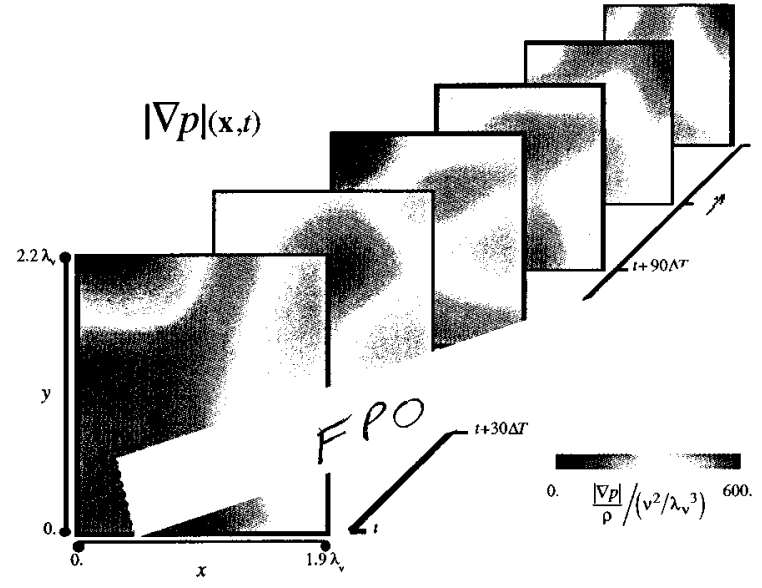

FIG. 29. The pressure gradient magnitude field $|\nabla p|(\mathbf{x}, t)$ for the same series of six planes used in Figs. 11, 12, 14, 16, 18, and 21. In addition to the components of $\mathbf{u}$ and $\nabla \mathbf{u}$ shown in those figures, determination of $\nabla p$ requires the Laplacian of each of the velocity components as well as their time derivatives, in accordance with the Navier-Stokes equations.

Figure 28 shows the results obtained for the degree of alignment of the scalar gradient vector with each of the three principal axes of the local strain rate tensor. These are presented as distributions of $\left|\cos \theta_{i}\right|$, where $\theta_{1}$ is the angle between $\nabla \zeta$ and the most extensional principal strain rate axis, and $\theta_{2}$ and $\theta_{3}$ are the angles between $\nabla \zeta$ and the intermediate and most compressional principal axes, respectively. The strong tendency toward alignment of the scalar gradient vector with the most compressional strain rate eigenvector (namely $\left|\cos \theta_{3}\right| \rightarrow 1$ ) is evident. The tendency of $\nabla \zeta$ to be orthogonal to the most extensional and the intermediate strain rate eigenvectors $\left(\left|\cos \theta_{1,2}\right| \rightarrow 0\right)$ is also apparent. There appears to be no difference in the alignment of $\nabla \zeta$ with either of these two eigenvectors. The bottom panel in this figure presents the distributions of $\left|\cos \theta_{3}\right|$ for those points where the scalar gradient magnitude, expressed as the normalized scalar energy dissipation rate $\nabla \zeta \cdot \nabla \zeta /\left(\langle\zeta\rangle / \lambda_{D}\right)^{2}$, exceeds the threshold values indicated. It is evident that higher values of scalar energy dissipation are associated with better alignment with the most compressive strain rate axis, consistent with the earlier results of Ashurst et al. ${ }^{19}$ from numerical simulations. The present results appear to represent the first experimental measurements of the alignment of scalar gradients with strain rate eigenvectors in turbulent flows.

\section{Pressure gradient fields}

The preceding sections have presented results related to the spatial structure of the velocity gradient fields $\nabla \mathbf{u}(\mathbf{x}, t)$ obtained with the present scalar imaging velocimetry technique in turbulent flows. However, since these SIV measurements inherently yield the fully resolved temporal information in these velocity fields as well, the results obtained provide access to certain dynamical quantities which have proven largely intractable to previous experimental studies. Perhaps the most prominent among these is the pressure gra- dient field $\nabla p(\mathbf{x}, t)$, determination of which requires simultaneous spatial and temporal differentiation of the velocity field. From the Navier-Stokes equations, the pressure gradient field can be obtained as

$$
\nabla p(\mathbf{x}, t)=-\left[\frac{\partial}{\partial t}+\mathbf{u} \cdot \nabla-\frac{1}{\operatorname{Re}} \nabla^{2}\right] \mathbf{u}(\mathbf{x}, t),
$$

where, for the present incompressible case, the density $\rho$ has been absorbed into the pressure. Determination of pressure gradient fields and their statistics has been a key objective in aeroacoustics, yet the associated need for fully resolved, four-dimensional vector velocity fields has, to date, made measurement of these fields impossible. The present scalar imaging velocimetry measurements allow the true pressure gradient fields to be determined. Examples of the velocity and velocity gradient field terms appearing in Eq. (22) were shown in previous sections. Additionally, Eq. (22) requires both time derivatives and the Laplacians of the measured velocity fields, which are found by direct differentiation on the four-dimensional velocity field data volumes. Figure 29 shows the resulting pressure gradient magnitude $|\nabla p|(\mathbf{x}, t)$ for the same series of six planes for which the velocity gradient fields were presented in the previous sections. While an extensive investigation of the pressure gradient field in turbulent flows is well beyond the scope of the present work, these results demonstrate the experimental extraction of such information in a turbulent flow using scalar imaging velocimetry.

\section{DISCUSSION AND CONCLUSIONS}

This paper has demonstrated practical application of a new approach to fluid velocimetry, developed and validated in Refs. 1 and 2, that allows the fully resolved, space- and time-varying vector velocity field $\mathbf{u}(\mathbf{x}, t)$ to be determined in turbulent flows from measurements of a single, dynamically passive, conserved scalar field $\zeta(\mathbf{x}, t)$. This scalar imaging velocimetry technique has been applied to experimental scalar field data to obtain the first non-invasive measurements of the full velocity gradient tensor $\nabla \mathbf{u}(\mathbf{x}, t)$ at the small scales of a turbulent flow. The results obtained provide a level of detailed access to the structure and dynamics of real, inhomogeneous, anisotropic turbulent shear flows that to date has been realizable only by direct numerical simulations (DNS) of turbulence under idealized conditions.

The scalar imaging velocimetry method is based on inversion of the exact transport equation (7) which governs the evolution of the conserved scalar field, together with the physical constraint $\lambda_{\nu}=\lambda_{D} \cdot \mathrm{Sc}^{1 / 2}$ on the relative smoothness of the scalar and velocity fields in turbulent flows. The scalar transport equation ensures that the resulting velocity field $\mathbf{u}(\mathbf{x}, t)$ is consistent with the scalar field data $\zeta(\mathbf{x}, t)$, while the smoothness condition excludes all spurious velocity fields that would be admissible by the scalar transport equation alone. These spurious fields arise from the form of the advective term in Eq. (7), which allows any velocity field having streamlines confined to isoscalar surfaces to be added to the true velocity field without affecting the scalar field evolution. Such spurious velocity fields must contain length 
scales as small as those in the scalar field data. However, for $\mathrm{Sc}>1$ this is inconsistent with the physical requirement on the smallest length scale ratio that can be sustained by the strain-diffusion balance in turbulent flows. Imposing this length scale requirement as a smoothness condition on the scalar and velocity fields therefore excludes the spurious additive velocity fields, and allows for unique determination of the velocity field from scalar field data.

Inversion of the scalar transport equation to yield the velocity field from scalar field data can be performed directly, as was shown in Ref. 1 , where the smoothness constraint was imposed implicitly. This inversion can alternatively be done through a variational formulation, as presented in Part I, in which the smoothness constraint is imposed explicitly and which is inherently less sensitive to noise and other errors in the scalar field data. It was shown through DNS-based validation tests ${ }^{2}$ that the variational formulation gives more accurate results than does the direct inversion formulation. In absolute terms, the velocity fields extracted from scalar field data using the variational formulation show better than $95 \%$ correlations with the true DNS results. It must be kept in mind that the results from these DNS tests were obtained in the limiting case of $\mathrm{Sc}=1$ scalar field data, for which the original scalar field contains nominally the same amount of information as does the objective velocity field. In contrast, for the $\mathrm{Sc} \gg 1$ scalar field data used here, the velocity field sought contains less information than does the scalar field, since for those data $\left(\lambda_{\nu} / \lambda_{D}\right)=\mathrm{Sc}^{1 / 2} \approx 45$. Thus the $\mathrm{Sc} \gg 1$ scalar field data carry redundant information for the purpose of determining the velocity field. It can be expected that, all other things being equal, this large redundancy of information leads to velocity field results that are at least as accurate as those obtained in the $\mathrm{Sc}=1 \mathrm{DNS}$ validation tests.

Here the scalar imaging velocimetry technique has been applied to fully resolved, four-dimensional scalar field data (with $\mathrm{Sc} \approx 2075$ ) of the type described in Refs. 3, 4, and 5 to yield the first, non-invasive, spatio-temporal measurements of the full velocity gradient tensor at the small scales of a turbulent flow. These data were obtained in the fully developed, self-similar far field of an axisymmetric turbulent jet, at an axial location 235 diameters downstream of the jet exit. The outer scale Reynolds numbers were in the range $3,000 \leqslant \operatorname{Re}_{\delta} \leqslant 4,200$, with Taylor scale Reynolds numbers $\operatorname{Re}_{\lambda} \approx 45$. The scalar field data in each of three cases considered contain over 3 billion individual point measurements of the local conserved scalar value, having spatial separations $(\Delta x, \Delta y, \Delta z)<0.5 \lambda_{D}$ and temporal separations $\Delta T \leqslant 0.5 \lambda_{D} / U$. The high signal-to-noise ratio of these measurements allows accurate determination of the scalar field derivatives $\partial \zeta(\mathbf{x}, t) / \partial t, \nabla \zeta(\mathbf{x}, t)$ and $\nabla^{2} \zeta(\mathbf{x}, t)$, which provide the inputs to the SIV technique.

Samples of the spatio-temporal results obtained from these scalar imaging velocimetry measurements have been presented for the complete velocity $\mathbf{u}(\mathbf{x}, t)$ and velocity gradient $\nabla \mathbf{u}(\mathbf{x}, t)$ fields at the small scales of a turbulent flow in Figs. 9-12, 14, 16, and 18. The availability of the full ninecomponent velocity gradient tensor allows all three components of the vector vorticity field $\omega(\mathbf{x}, t)$ and all six compo- nents of the normal and shear strain rate fields $\varepsilon(\mathbf{x}, t)$ to be determined. Owing to the very high Schmidt number at which these measurements are made, the spatial planes typically span about two inner length scales $\lambda_{\nu}$ in each direction, giving the inner scale structure of the various fields shown. This limited range of accessible spatial length scales allows determination of spatial structure, spatial spectra, and other information deep within the dissipative scales of motion, but precludes spatial information on larger scales. However, the much longer scaled temporal dimension of the resulting data, which typically spans well over 75 advective time scales $\lambda_{\nu} / U$, allows access to temporal structure and spectra extending into the inertial range of scales. Additionally, the combined four-dimensional data allow probability distributions of the $\nabla \mathbf{u}(\mathbf{x}, t)$ components to be determined which have essentially converged to their statistically stationary form. The corresponding probability distributions have been presented for the symmetric and anti-symmetric parts of the complete velocity gradient tensor field $\nabla \mathbf{u}(\mathbf{x}, t)$ at the small scales of a turbulent flow in Figs. 15, 17 and 19.

There are several features of these results which are of particular interest, both in themselves, as well as for comparison with previous laboratory measurements and results of direct numerical simulations. Such comparisons serve as partial validation of the present scalar imaging velocimetry results.

When normalized by the inner reference scales $\lambda_{\nu}$ and $\nu$, the probability distributions for the three data sets considered are substantially in agreement. Although the Reynolds numbers involved do not differ widely, one of the three cases appeared to involve significant buoyancy effects; however, after a rescaling of the length scale $\lambda_{\nu}$ the results obtained for that case yield essentially the same probability distributions as do the non-buoyant jet cases. This can be seen in Figs. 15, 17 and 19, and even more dramatically in the distributions obtained for higher-order velocity gradient quantities in Fig. 20.

The exponential scaling in the tails of these distributions agrees with results obtained at much higher Reynolds numbers and in fundamentally different turbulent flows, from both experiments and direct numerical simulations.

The zero-divergence requirement from continuity appears to be well satisfied by these results, with the 0.73 correlation between $(\partial u / \partial x)$ and $(-\partial v / \partial y-\partial w / \partial z)$ comparing favorably with the values 0.25 to 0.7 reported in invasive multi-probe measurements. ${ }^{12}$ This relatively good agreement with the incompressibility condition results in part from its having been included explicitly in the variational inversion, however the weight assigned to it is smaller than that assigned to the scalar transport equation.

Results obtained for increasingly high-order velocity gradient quantities show increasing levels of internal intermittency. Second-order quantities such as the kinetic energy dissipation rate field $\Phi(\mathbf{x}, t) \equiv(2 / \operatorname{Re}) \varepsilon: \varepsilon$ and the enstrophy field $\frac{1}{2} \omega \cdot \omega(\mathbf{x}, t)$ in Figs. 20 and 21 are "spotty,", with large values being very infrequent. Third-order quantities such as the enstrophy production rate field $\omega \cdot \varepsilon \cdot \omega(\mathbf{x}, t)$ in Figs. 20 and 21 show even higher internal intermittency. The intermittency in the dissipation rate field leads to inertial range 
structure function exponents (Fig. 23) that agree well with previous measurements by Anselmet et al. ${ }^{14}$ at Taylor scale Reynolds numbers $515 \leqslant \operatorname{Re}_{\lambda_{T}} \leqslant 852$, in both turbulent jet and turbulent duct flows.

The alignments observed in Figs. 26 and 28 for both the vorticity vector field $\omega(\mathbf{x}, t)$ and the scalar gradient field $\zeta(\mathbf{x}, t)$ with the strain rate tensor eigenvectors $\left(\hat{e}_{\varepsilon_{11}}, \hat{e}_{\varepsilon_{22}}, \hat{e}_{\varepsilon_{33}}\right)$ agree with previous DNS results in homogeneous, isotropic turbulence at higher $\operatorname{Re}_{\lambda_{T}}$ values, as does the present lack of any strong alignment between the velocity vector field $\mathbf{u}(\mathbf{x}, t)$ and the vorticity vector field $\omega(\mathbf{x}, t)$ seen in the helicity distributions of Fig. 27 and the positivity of the intermediate strain rate $\varepsilon_{22}$ in Fig. 25. Further, the present results show improved alignment of the scalar gradient vector with the most compressive principal strain rate axis for increasing scalar gradient vector magnitudes, which is also in agreement with DNS results at higher Reynolds numbers and in fundamentally different turbulent flows. The present results also provide the first experimental measurements of the full vector pressure gradient field $\nabla p(\mathbf{x}, t)$ in a turbulent flow.

Collectively, the present scalar imaging velocimetry results, obtained on the inner scales of a turbulent flow, are generally in good agreement with both direct numerical simulation results and experimental results obtained at much larger Reynolds numbers and in fundamentally different flows. This suggests that the present outer scale Reynolds numbers $3,000 \leqslant \operatorname{Re}_{\delta} \leqslant 4,200$ and corresponding Taylor scale Reynolds numbers $41 \leqslant \operatorname{Re}_{\lambda_{T}} \leqslant 48$ are sufficiently large for the presumed quasi-universality of the small scales to have been approached, so that the results, when scaled by inner variables, will show only a weak remaining dependence on both the Reynolds number and the particular flow in which the measurements were made. Although these Reynolds numbers may seem relatively low in comparison with traditional turbulence studies, there is considerable evidence in the literature that the physical structure of the small scales in turbulent flows establishes itself well before many of the traditional hallmarks of high Reynolds number turbulence are reached. For example, experimental evidence (e.g., Dowling $^{24}$ ) shows that the small scale portion of Kolmogorov-normalized scalar power spectra collapse for outer scale Reynolds numbers from 5,000 to 40,000, even though no inertial range with a $k^{-5 / 3}$ scaling exists for the lower Reynolds numbers. These "hallmarks" (such as an extensive $k^{-5 / 3}$ range) are thus believed to be signatures of this fundamental small scale structure that manifest themselves once sufficiently high Reynolds numbers are reached, rather than being minimum requirements necessary even to achieve this fundamental small scale structure. Recent evidence from direct numerical simulations of turbulent flows appears to support this view. In DNS studies with $35 \leqslant \operatorname{Re}_{\lambda_{T}} \leqslant 170$, Jiménez, Wray, Saffman and Rogallo ${ }^{11}$ find essentially perfect collapse of small scale spectra (see their Figs. 1a and 2a) as well as small scale vortical structure (see their Figs. 11a,b), and, regarding the small scale structure of the flow, state "it is surprising that no obvious increase in complication is detected as $\operatorname{Re}_{\lambda_{T}}$ increases." They conclude that "it is surprising that we are able to find similarity laws spanning the whole range of Reynolds numbers, and that even the lowest- $\mathrm{Re}_{\lambda_{T}}$ flow seems to be essentially turbulent." This suggests that the Taylor scale Reynolds numbers accessible in the present measurements are large enough for the small scale structure to approach a Reynolds number asymptotic state. As a consequence, the results obtained here are believed to be largely representative of the quasiuniversal small scale structure of all high Reynolds number turbulent flows.

\section{ACKNOWLEDGMENTS}

The first author was supported by a François-Xavier Bagnoud Fellowship from the Association FXB. The scalar field measurements used in this study were obtained as part of the dissertation work of Dr. Kenneth B. Southerland at Michigan under Air Force Office of Scientific Research (AFOSR) Grants No. 89-0541 and F49620-95-J-0115, and Gas Research Institute (GRI) Contracts No. 5087-260-1443 and 5093-260-2698.

${ }^{1}$ W. J. A. Dahm, L. K. Su, and K. B. Southerland, “A scalar imaging velocimetry technique for fully resolved four-dimensional vector velocity field measurements in turbulent flows," Phys. Fluids A 4, 2191 (1992).

${ }^{2}$ L. K. Su and W. J. A. Dahm, "Scalar imaging velocimetry measurements of the velocity gradient tensor field in turbulent flows. I. Assessment of errors," Phys. Fluids 8, 1869 (1996).

${ }^{3}$ K. B. Southerland, "A four-dimensional experimental study of conserved scalar mixing in turbulent flows," Ph.D. thesis, The University of Michigan, Ann Arbor, Michigan, 1994.

${ }^{4}$ K. B. Southerland and W. J. A. Dahm, "A four-dimensional experimental study of conserved scalar mixing in turbulent flows," Report No. 02677912, Department of Aerospace Engineering, The University of Michigan, Ann Arbor, Michigan, 1994; submitted to J. Fluid Mech.

${ }^{5}$ W. J. A. Dahm, K. B. Southerland, and K. A. Buch, "Direct, highresolution, four-dimensional measurements of the fine scale structure of $S c \gg 1$ molecular mixing in turbulent flows," Phys. Fluids A 3, 1115 (1991).

${ }^{6} \mathrm{~K}$. A. Buch, "Fine scale structure of conserved scalar mixing in turbulent shear flows: $S c \gg 1, S c \approx 1$ and implications for reacting flows," Ph.D. thesis, The University of Michigan, Ann Arbor, Michigan, 1991.

${ }^{7}$ A. N. Tikhonov and V. Y. Arsenin, Solutions of Ill-Posed Problems (Winston, Washington, D.C., 1977).

${ }^{8}$ P. R. Bevington and D. K. Robinson, Data Reduction and Error Analysis for the Physical Sciences (McGraw-Hill, New York, 1992).

${ }^{9}$ L. K. Su, "Scalar imaging velocimetry: Development and application in measurements of fine structure and dynamics in turbulent flows," Ph.D. thesis, The University of Michigan, Ann Arbor, Michigan, 1995.

${ }^{10}$ L. K. Su and W. J. A. Dahm, "Scalar imaging velocimetry and its application in measurements of the structure and dynamics of the complete velocity gradient tensor in turbulent flows," Report No. 026779-14, Department of Aerospace Engineering, The University of Michigan, Ann Arbor, Michigan, 1995.

${ }^{11}$ J. Jiménez, A. A. Wray, P. G. Saffman, and R. S. Rogallo, "The structure of intense vorticity in isotropic turbulence," J. Fluid Mech. 255, 65 (1993).

${ }^{12}$ A. Tsinober, E. Kit, and T. Dracos, "Experimental investigation of the field of velocity gradients in turbulent flows," J. Fluid Mech. 242, 169 (1992)

${ }^{13} \mathrm{G}$. K. Batchelor and A. A. Townsend, "The nature of turbulent motion at large wavenumbers,' Proc. R. Soc. London Ser. A 199, 238 (1949).

${ }^{14}$ F. Anselmet, Y. Gagne, E. J. Hopfinger, and R. A. Antonia, "High-order velocity structure functions in turbulent shear flows," J. Fluid Mech. 140, 63 (1984).

${ }^{15}$ A. N. Kolmogorov, "A refinement of previous hypotheses concerning the local structure of turbulence in viscous incompressible fluid at high Reynolds number,' J. Fluid Mech. 13, 82 (1962). 
${ }^{16}$ U. Frisch, "From global scaling, á la Kolmogorov, to local multifractal scaling in fully developed turbulence,' Proc. R. Soc. London Ser. A 434, 89 (1991).

${ }^{17}$ M. M. Rogers and P. Moin, "The structure of the velocity field in homogeneous turbulent flows,', J. Fluid Mech. 176, 33 (1987).

${ }^{18}$ A. N. Kolmogorov, "Local structure of turbulence in an incompressible fluid at very high Reynolds numbers,', C.R. Acad. Sci. URSS 30, 301 (1941).

${ }^{19}$ W. T. Ashurst, A. R. Kerstein, R. M. Kerr, and C. H. Gibson, “Alignment of vorticity and scalar gradient in simulated Navier-Stokes turbulence,', Phys. Fluids 30, 2343 (1987).

${ }^{20}$ Z.-S. She, E. Jackson, and S. A. Orszag, "Structure and dynamics of homogeneous turbulence: models and simulations,', Proc. R. Soc. London Ser. A 434, 101 (1991).

${ }^{21}$ M. M. Rogers and P. Moin, "Helicity fluctuations in incompressible turbulent flows,' Phys. Fluids 30, 2662 (1987).

${ }^{22}$ J. M. Wallace, J.-L. Balint, and L. Ong, "An experimental study of helicity density in turbulent flows,', Phys. Fluids A 4, 2013 (1992).

${ }^{23}$ K. A. Buch and W. J. A. Dahm, "Fine scale structure of conserved scalar mixing in turbulent shear flows: $S c \gg 1, S c \approx 1$, and implications for reacting flows,' Report No. 026779-5, Department of Aerospace Engineering, The University of Michigan, Ann Arbor, Michigan, 1991.

${ }^{24}$ D. R. Dowling, "The estimated scalar dissipation rate in gas-phase turbulent jets,' Phys. Fluids A 3, 2229 (1991). 